Elsevier required licence: (C) <2021>. This manuscript version is made available under the CC-BY-NCND 4.0 license http://creativecommons.org/licenses/by-nc-nd/4.0/

The definitive publisher version is available online at

[https://www.sciencedirect.com/science/article/abs/pii/S2214391221000714?via\%3Dihub] 


\title{
Dynamic Parameters of Subgrade Soils Prone to Mud Pumping Considering the Influence of Kaolin Content and the Cyclic Stress Ratio
}

Nguyen, T.T., Indraratna, B., and Singh, M. 2021. Dynamic Parameters of Subgrade Soils Prone to Mud Pumping Considering the Influence of Kaolin Content and the Cyclic Stress Ratio. Transportation Geotechnics: 100581. doi: https://doi.org/10.1016/j.trgeo.2021.100581.

\begin{abstract}
Rail transport industry often desires to increase the freight capacities for heavy-haul trains to transport more goods. However, this requires an increase in the axle loads, which results in the railway foundation being subjected to higher cyclic stress ratio (CSR), causing significant subgrade distress. Recent laboratory investigations indicate that addition of kaolin to low-tomedium plasticity soils can avert early softening and fluidization of the soil under increasing $C S R$, how the dynamic parameters such as the shear modulus $(G)$ and the damping ratio $\left(D_{p}\right)$ of soils may change consequentially to this modification needs appropriate quantification. Furthermore, while there are plenty of studies correlating shear strain and plasticity index $(P I)$ with the dynamic properties of the soil, the role of loading parameters such as the CSR and number of cycles $(N)$ has not been thoroughly investigated. Therefore, efforts have been made to characterise the dynamic parameters of moderately plastic silty-clay soils with varying kaolin content $\left(c_{K}\right)$ and loading parameters. The results indicate that as CSR exceeds a critical level, $G$ decreases rapidly whereas $D_{p}$ was found to increase abruptly in few loading cycles. The paper also shows that when $G$ diminishes about $20 \%$ from its initial value, any further loss in shear modulus would cause soil to turn into an unstable stage where the excess pore water pressure and shear strain begins to increase rapidly. Although varying the kaolin content does not alter the PI significantly, it causes a considerable effect on the dynamic parameters of the soil. Based on the current experimental findings, empirical models are proposed to estimate dynamic parameters of subgrade soils prone to mud pumping considering loading condition under heavy haul railways.
\end{abstract}

Keywords: subgrade soil, damping ratio, shear wave velocity, shear modulus, degradation 


\section{List of Abbreviations and Notation}

\begin{tabular}{|c|c|}
\hline $\mathrm{BE}$ & Bender Element \\
\hline$C S R$ & Cyclic Stress Ratio \\
\hline$C S R_{c}$ & Critical threshold of CSR \\
\hline CTT & Cyclic Triaxial Test \\
\hline$c_{K}$ & Content of Kaolin \\
\hline$D_{p}$ & Damping Ratio \\
\hline EPWP & Excess Pore water Pressure \\
\hline$e$ & Void ratio \\
\hline$f$ & Frequency \\
\hline$G$ & Shear Modulus \\
\hline$G^{b}$ & Shear modulus as determined by bender element test (small strain) \\
\hline$G^{c}$ & Shear modulus as determined by cyclic triaxial test (large strain) \\
\hline$L_{t}$ & Length that the signal travels \\
\hline$N$ & Number of cycles \\
\hline$N_{c}$ & Critical number of cycles \\
\hline$V_{s}$ & Shear wave velocity \\
\hline$\varepsilon_{s}$ & Shear strain \\
\hline$\lambda$ & Wave length \\
\hline$\rho$ & Bulk (saturated) density of soil \\
\hline$\rho_{d}$ & Dry density of soil \\
\hline$v$ & Poisson's ratio \\
\hline
\end{tabular}




\section{Introduction}

As the train passes over a railway foundation, it creates a complex stress path under the rail tracks which is dependent on several factors such as the axle load, the speed of moving train, the train characteristics (e.g., the effective length between wheels), and properties of the track foundation (Gräbe and Clayton 2009; Dareeju et al. 2017; Nguyen et al. 2019). Despite this complexity, it is apparent that the train load propagates through the underlying system in which the key dynamic parameters such as the shear wave velocity $\left(V_{s}\right)$, damping ratio $\left(D_{p}\right)$, shear modulus $(G)$ and Poisson's ratio (v) would mainly govern the process (Kramer 1996). While $V_{s}$ represents how fast a given shear wave can propagate in a medium, $D_{p}$ measures how the dynamic excitation dissipates over time. Higher $D_{p}$ represents more amount of kinetic energy being transferred to other forms such as heat, friction and plasticity yielding. $G$ and $v$ describe the variation of shear strain with respect to the loading characteristics (i.e., stress state). Therefore, proper determination and understanding of these dynamic parameters play a crucial role in the accuracy of any analysis pertaining to railway foundation (Fig. 1).

The behaviour of shear modulus and damping ratio, which are associated with the energy dissipation of subgrade soils under cyclic loads, has received considerable attention over the past years (Vucetic and Dobry 1991; Kallioglou et al. 2008; Cai et al. 2018; Nguyen and Indraratna 2020b), albeit still limited data considering heavy railway conditions such as low confining pressure $\left(\sigma_{c}\right)$ and large cyclic stress ratio (CSR) (Indraratna et al. 2020b). Previous studies indicate that the dynamic parameters of subgrade soils are strongly dependent on the confining pressure. For example, at smaller confining pressures, there is an earlier inception of shear modulus degradation accompanied by larger damping (Sun et al. 1988; Kallioglou et al. 2008). The influence of confining pressure on the behaviour of $G$ and $D_{p}$ is more prominent for soils with low to medium plasticity, which are highly susceptible to 
subgrade fluidization or mud pumping (Nguyen et al. 2019; Nguyen and Indraratna 2021). While most of the previous studies (Sun et al. 1988; Vucetic and Dobry 1991; Ichii and Mikami 2018) normally address these issues at relatively low frequency range $(<1.0 \mathrm{~Hz})$ considering seismic perspective, these cannot be applied directly to railway loading that are often subjected to higher loading frequencies $(>1.0 \mathrm{~Hz})$.

The kinetic energy transformation and dissipation is strongly governed by the internal friction and plasticity characteristics of materials (Vucetic et al. 1998a; Nguyen and Indraratna 2020b; Truong et al. 2021). Therefore, the properties of subgrade soil, such as the density (or void ratio), plasticity index (PI) and fines content, certainly affect how the soil foundation can dampen the cyclic excitation. In fact, past experimental studies have shown significant influence of void ratio and PI on the behaviour of $G$ and $D_{p}$ (Vucetic and Dobry 1991; Kallioglou et al. 2008). For example, soils with high PI experience less degradation in shear modulus but more damping with increasing shear strain. Recent studies (Kim et al. 2016; Mamou et al. 2017b; Indraratna et al. 2020a) have indicated that varying fines content can have considerable influence on the cyclic behaviour of soils, how this can affect the dynamic parameters of soils needs further investigation.

Recent investigation based on cyclic triaxial tests (Indraratna et al. 2020a) revealed that increasing $C S R$ and $N$ at low confining pressure (i.e., 15 to $20 \mathrm{kPa}$ ) can cause the soil specimen to soften, which is accompanied by a rapid increase in the excess pore water pressure and shear strain. Further, the effect of kaolin (fines) content $\left(c_{K}\right)$ and soil density on the cyclic response of subgrade soil, especially in mitigating the build-up of excess pore pressure and fluidization of the soil, was also captured. However, due to the limited scope of their studies, the results and corresponding discussion were restrained mainly to conventional soil parameters such as the excess pore pressure, stress and strain components while the dynamic parameters had not been evaluated. Therefore, this paper aims to extend the 
previous results and discussion to explain the behaviour of dynamic parameters of the soil by considering varying loading parameters and kaolin content. The study concentrates on shallow low to medium plasticity subgrade soils which are most vulnerable to mud pumping under heavy haul railways. Indeed, there is a lack of effort to investigate how loading characteristics such as increasing CSR, and kaolin content can affect dynamic properties of soils (Vucetic and Dobry 1991; Vucetic et al. 1998a; Kallioglou et al. 2008), which is why the current study becomes crucial. In addition to the experimental data adopted from Indraratna et al. (2020a), bender element (BE) tests are also carried out to address dynamic behaviour of the subgrade soil. Moreover, because the Poisson's ratio is usually less varying with loading properties, the current paper mainly focuses on the behaviour of $V_{s}, G$ and $D_{p}$.

\section{Laboratory testing and analysis method}

\subsection{Cyclic Triaxial Test}

A series of cyclic triaxial testing (CTT) were carried out on a silty clayey soil compacted at different dry densities, i.e., 1620,1706 and $1810 \mathrm{~kg} / \mathrm{m}^{3}$. Additionally, 10 and $30 \%$ by mass of kaolin was also added to the soil to examine how kaolin content would affect its cyclic behaviour. A low initial effective confining pressure (i.e., $20 \mathrm{kPa}$ ) was applied to fully saturated test specimens. Further, the specimens were subjected to varying $\operatorname{CSR}(0.2$ to 1.2$)$ under undrained condition. The results indicate that subgrade soil experiences an early softening (i.e., the shear strength drops at early stage of loading), accompanied with a rapid increase in excess pore water pressure and axial strain when the CSR exceeds a certain critical level $\left(C S R_{c}\right)$, whereas the soil remains stable for over 50,000 cycles when $C S R<$ $C S R_{c}$. To supplement the cyclic triaxial test data, a series of bender element (BE) tests was performed to address the shear wave propagation and the associated shear modulus $G$ at small strain, which is detailed in the following section. 


\subsection{Bender element test}

Bender element tests have been widely used to estimate the shear wave velocity $V_{s}$ and shear modulus $G$ at small strains for different geomaterials (Viggiani and Atkinson 1995; Heitor et al. 2013; Ogino et al. 2015), so it was applied onto the subgrade soils used in the current study. The velocity $V_{s}$ was computed by dividing the length $\left(L_{t}\right)$ with the time $\left(t_{t}\right)$ that the signal traveled through the soil (i.e., $V_{s}=L_{t} / t_{t}$ ). The current study considered $L_{t}$ as the tip-totip distance between the two bender elements installed at both ends of the specimen. The first peak-to-peak arrival (see Fig. 2b) was used to determine the travel time with reference to previous studies (Viggiani and Atkinson 1995; Lee and Santamarina 2005; Ogino et al. 2015). The signal at different frequencies was sent and the results were evaluated to ensure the ratio between $L_{t}$ and the wave length $\lambda$ was kept over 2 (i.e., $L_{t} / \lambda>2$ ) to minimize the near-field effect (Arroyo et al. 2003; Wang et al. 2007). In this study, a frequency of $5 \mathrm{kHz}$ was found to satisfy this condition and was therefore applied to all specimens.

The soil specimens used for the current BE tests had the same size (i.e., $50 \mathrm{~mm}$ in diameter and $100 \mathrm{~mm}$ in length), dry density and kaolin content to those used in the triaxial test. The back pressure and cell pressures were increased to $380 \mathrm{kPa}$ and $400 \mathrm{kPa}$, respectively, to achieve the saturation condition, i.e., Skempton's B value $>0.95$ while the initial confining pressure of $20 \mathrm{kPa}$ and vertical stress of $33.33 \mathrm{kPa}$ (i.e., anisotropic condition with $\left.K_{o}=0.6\right)$ was applied. Fig. 2 a represents how the Bender elements were attached on the soil specimen, while Fig. $2 \mathrm{~b}$ shows examples of how the signal $(f=5 \mathrm{kHz})$ transmitted through the soil specimen. It is also noted that the signal intensity attenuates significantly when travelling through the soil, from 14 (peak input) to about $7.0 \mathrm{~V}$ (peak output). In this study, the GDSBES V.20 software incorporated in GDS Bender element system was used (GDS 2020). 


\subsection{Method to determine the dynamic parameters}

\section{Shear modulus $G$}

The current study employed two different approaches to estimate $G$ with respect to different levels of strain. The first method was based on the results from BE tests at very small strain levels (denoted as $G^{b}$ with shear strain $\varepsilon_{s}<10^{-5}$ ) (Vucetic and Dobry 1991; Kallioglou et al. 2008) where the soil behaviour could be considered as fully elastic. The second approach was based on the experimental results from cyclic triaxial test (CTT) data at higher levels of strain (denoted as $G^{c} ; 0.01 \%<\varepsilon_{s}<10 \%$ ). As $G$ tends to decrease with increasing strain, the

magnitude of $G$ at small strain (i.e., $G^{b}$ ) is considered to be the maximum value of $G$ (i.e., $\left.G_{\max }\right)$ for the soil. $G^{b}$ can be estimated by:

$$
G^{b}=\rho V_{s}^{2}
$$

where $\rho$ is the bulk (saturated) density of the soil.

In the second approach, the relationship between the deviator stress $q$ and the shear strain $\varepsilon_{s}$ obtained from CTT is adopted. Specifically, Fig. 3 presents how the shear modulus $G^{c}$ can be estimated from this data, noting that $\mathcal{E}_{s}$ can vary up to $10 \%$ in this approach. Theoretically, $G^{c}$ is the slope of the line connecting the initial point (or the origin in the first cycle) and the peak of the loading loop, which means that $G^{c}$ can decrease over the number of cycles because of soil degradation.

Damping ratio $D_{p}$

Damping ratio $D_{p}$ can be estimated based on the hysteresis loop generated due to cyclic loading (ASTM D3999 - 91 2003). Fig. 3b represents this concept where $D_{p}$ is defined as follows: 


$$
D_{p}=\frac{\Delta W}{4 \pi W}
$$

where $\Delta W$ and $W$ are the dissipated energy and the total energy, respectively, during a loading cycle. The ratio between $\Delta W$ and $W$ can be calculated as the ratio between the loop and triangle areas generated by the loading and unloading curves as shown in Fig. $3 b$. It is noteworthy that the dissipated area depends mainly on the plastic strain that remains after each loading cycle, which indicates how plastic properties of the subgrade soil can be used to mitigate the effect of cyclic excitation.

When the specimen becomes fully fluidized (i.e., fluid-like state), the specimen does not withstand any further load (i.e., almost zero shear strength), resulting in excessive deformation. The test was terminated automatically when the specimens reached an axial strain of $10 \%$ (Indraratna et al. 2020a). Furthermore, the analysis for $G$ and $D_{p}$ was carried out for up to 1000 cycles to reflect long and heavy trains (i.e., 2.5 to $7 \mathrm{~km}$ long and 25 to 40 tonnes axle load) which commonly operate in mining regions (Australian Trade Commission 2013; Railway Gazette International 2016).

\section{Results and Discussion}

\subsection{Shear wave velocity of subgrade soil}

\section{Effects of soil density (or void ratio)}

As the shear wave mainly propagates through the solid skeleton of the soil (note: specimens were fully saturated), it is understandable that the higher the degree of compaction the larger the shear wave velocity. Fig. 4 shows the variation of $V_{s}$ with dry density and kaolin content

$c_{K}$. In particular, the magnitude of $V_{s}$ increases from $184 \mathrm{~m} / \mathrm{s}$ at $\rho_{d}=1620 \mathrm{~kg} / \mathrm{m}^{3}$ to 204 and $225 \mathrm{~m} / \mathrm{s}$ as $\rho_{d}$ increases to 1710 and $1805 \mathrm{~kg} / \mathrm{m}^{3}$, respectively. The corresponding $G^{\mathrm{b}}$ for these 
specimens $\left(\rho_{d}=1620,1710\right.$ and $\left.1805 \mathrm{~kg} / \mathrm{m}^{3}\right)$ was computed (Eq. [1]) as 68.1, 85.9 and 107.6 $\mathrm{MPa}$, respectively. By combining the current data with those given in previous studies (Kulkarni et al. 2010; Long and Donohue 2010; Heitor et al. 2013; Ogino et al. 2015) where low to medium plasticity soils $(\mathrm{PI} \leq 32)$ were tested under low confining pressure, the relationship between $V_{s}$ and void ratio $e$ can be obtained (Fig. 4b). It is interesting to note that $V_{s}$ gradually increases when $e$ decreases from about 2.9 to 1.2 before a sharp increase occurs when $e<0.9$. Moreover, $V_{s}$ jumps from about $75 \mathrm{~m} / \mathrm{s}$ at $e=0.9$ to nearly $200 \mathrm{~m} / \mathrm{s}$ as $e$ decreases to around 0.55 . This indicates a certain threshold of void ratio that leads to a significant increment of $V_{s}$ and the associated shear strength $\left(G^{b}\right)$, for instance, Fig. $4 \mathrm{~b}$ shows this threshold of the void ratio to be about 0.9 for low to medium plasticity soil.

Shear modulus $G^{b}$ at small strain and the corresponding shear wave velocity $V_{s}$ can be computed based on an empirical relationship as follows (Kallioglou et al. 2008):

$$
G^{b}=A f(e)\left(\sigma_{0}^{\prime}\right)^{n}\left(p_{a}\right)^{1-n}
$$

where $f(e)$ is a function of void ratio that varies with different types of soil; $f(e)=e^{-1.1}$ is suggested for the clayed silty soil used in the current study; $p_{a}$ is the atmosphere pressure, i.e., $100 \mathrm{kPa}, A$ and $n$ are the empirical coefficients which are suggested at 506 and 0.42 , respectively for the current soil (D' Elia and Lanzo 1996); $\sigma_{o}^{\prime}$ is the initial effective stress. Given a small level of initial effective stress, i.e., 20-50 kPa at shallow subgrade, Eq. [3] can be simplified for shallow subgrade soil prone to mud pumping as follows:

$$
G^{b}(k P a)=(1.03 \sim 1.52) \times 10^{5} e^{-1.1}
$$

The above relationship also means that the corresponding $V_{s}$ can be simply estimated by, i.e., $V_{s}=(76 \sim 98) e^{-1.1}$ for a dry density from 1620 to $1805 \mathrm{~kg} / \mathrm{m}^{3}$. The larger the confining pressure, the larger the $V_{s}$. This relationship agrees well with the current and past experimental data shown in Fig. 4b, which hence suggests the simplified form (Eq. [4]) can 
be used to determine the maximum shear modulus of shallow low to medium plasticity (PI < 32) subgrade soils. The results also indicate that for soils with PI less than around 30, the effect of PI on the shear modulus can be negligible.

\section{Effect of kaolin content}

Inclusion of kaolin fines into the subgrade soil matrix reduces the value of $V_{s}$; for example, it decreases from $184 \mathrm{~m} / \mathrm{s}$ to 177 and $162 \mathrm{~m} / \mathrm{s}$ with $c_{K}$ of 10 and $30 \%$, respectively. This change of $V_{s}$ can be explained by a reduction in the average size of soil particles when $c_{K}$ increases in the soil. In particular, $D_{50}$ (i.e., the particle size finer than $50 \%$ by mass) of the subgrade soil decreases from $0.05 \mathrm{~mm}$ in the original soil to $0.022 \mathrm{~mm}$ and $0.013 \mathrm{~mm}$ in $\mathrm{K} 10$ and $\mathrm{K} 30$ soils, respectively (see Table 1). In other words, the larger the $D_{50}$, the larger the $V_{s}$. Previous studies (Kokusho and Yoshida 1997; KarrayMourad et al. 2011; Hussien and Karray 2015) also reveal a significant contribution of $D_{50}$ on the value of $V_{s}$, however, majority of these findings focus on granular soils $\left(D_{50}>0.1 \mathrm{~mm}\right)$. The current study provides a valuable addition to understand the influence of $D_{50}$ on $V_{s}$ in silty clay soils. Note that all soils were tested under the same condition of saturation and loading, thus only the effects of dry density (or void ratio) and fines content are addressed in the analysis.

\subsection{Degradation of subgrade shear modulus}

The value of shear modulus $G$ based on the data of cyclic triaxial tests (i.e., $G^{c}$ ) was estimated over different specimens using the approach shown in Fig. 3a. In order to represent the degradation of the shear modulus with the application of cyclic load, the ratio between the $G^{c}$ at a certain cycle $N$ and the initial value (i.e., $G_{o}^{c}$ obtained at the first loading cycle) where the value of $G^{c}$ is the largest (i.e., the degradation index $G^{c} / G_{o}^{c}$ ) was computed. Unlike conventional investigations where $G^{c} / G_{o}^{c}$ is considered in light of shear strain, the current

study concentrates on the influence of loading parameters including CSR and $N$ on $G^{c} / G_{o}^{c}$ 
associated with other factors such as soil density and kaolin content. Moreover, the current study addresses the dynamic behaviour of subgrade soil $(\mathrm{PI}=11-15)$ prone to mud pumping under rail tracks, other influencing factors such as PI, initial effective stress and over consolidation ratio (OCR) which have been studied well in past studies (Vucetic and Dobry 1991; Vucetic et al. 1998a; Kallioglou et al. 2008), are not further addressed in this paper.

\section{Influence of CSR and soil density}

Fig. 5 shows the variation of $G^{c}$ with $\mathrm{N}$ at different levels of $C S R$ for specimens compacted at different initial densities. The results show that there is negligible reduction in $G^{c}$ for stable specimens, i.e., $C S R<C S R_{c}$, whereas $G^{c}$ apparently decreases for $C S R \geq C S R_{c}$. Specifically, for $C S R=0.2$ where all the test specimens are stable, $G^{c}$ can maintain more than $93 \%$ of its initial value despite the increasing number of cycles, $N$. For this case, $\varepsilon_{s}$ reaches up to about $0.2 \%$ at the end of the test. When CSR exceeds the critical level, for instance 0.4 and 1.0 for specimen densities of 1620 and $1805 \mathrm{~kg} / \mathrm{m}^{3}$, respectively, $G^{c}$ begins to drop drastically. $G^{c}$ tends to lose more than $30 \%$ of its value when $N$ becomes larger than the critical number $N_{c}$, where the excess pore water pressure and shear strain begin to increase rapidly (Indraratna et al. 2020a). Clearly, the larger the $C S R$, the faster the degradation of $G^{c}$ as $N$ increases. It is important to note that some previous studies (Rollins et al. 1998; Vucetic et al. 1998b; Kallioglou et al. 2008) have estimated the degradation index with respect to the value of $G^{b}$ at small strain (i.e., $G_{\max }$ ), thus resulting in different rates of degradation compared to the current study where the largest value of $G$ during CTT data was considered.

To evaluate the effect of soil density, the values of $G^{c}$ estimated at different density specimens are combined in the same plot (i.e., Fig. 6), noting that only common $C S R$ values (i.e., 0.2-0.4) across different specimens are considered. Given the same number of cycles and $C S R$, it is evident that the smaller the void ratio, the greater the resistance to shear 
modulus degradation. For example, Fig. 6a shows that for $N=50$ cycles and $C S R=0.3$, while the specimen with a dry density $1620 \mathrm{~kg} / \mathrm{m}^{3}$ loses about $25 \%$ its initial shear strength, the test specimen with the larger density, i.e., $1710 \mathrm{~kg} / \mathrm{m}^{3}$ experiences only about $8 \%$ reduction in $G^{c}$. Interestingly, the specimen having the largest density $\left(1805 \mathrm{~kg} / \mathrm{m}^{3}\right)$ maintains almost a constant level of $G^{c}$ for the same loading parameters.

Fig. $6 \mathrm{~b}$ in which the behaviour of $G^{c}$ is considered at different levels of shear strain $\mathcal{E}_{s}$, shows the predominant role of $\mathcal{E}_{s}$ over the soil density (or void ratio) in governing the degradation of $G^{c}$. For instance, despite having different densities, $G^{c}$ begins to decrease when $\varepsilon_{s}$ exceeds $0.4 \%$ in all specimens. The larger the $\varepsilon_{s}$, the faster the degradation in $G^{c}$. This indicates that there is a critical threshold of $\varepsilon_{S}$ (i.e., $0.4 \%$ in this current data) which triggers a swift degradation in cyclic shear resistance of the soil. This finding is consistent with the results of Kallioglou et al. (2008) for a similar low plasticity soil (i.e., PI = 13 and void ratio, $e$ varying from 0.378 to 0.64$)$. Few other studies such as Sun et al. (1988) and Vucetic and Dobry (1991), also indicate the prominence of void ratio in the way that the shear strain can affect the degradation of $G^{c}$.

It is also interesting to note that the critical number of cycles $N_{c}$ (i.e., the red dot shown in Fig. 6b) where the excess pore water pressure (EPWP) and shear strain begin to develop rapidly, seems to occur when the loss of $G^{c}$ exceeds $20 \%$. For example, Fig. $6 \mathrm{~b}$ represents the variation of normalized shear modulus for all tested specimens with different values of density and $C S R$. It is noted that these specimens reach $N_{c}$ at approximately the same ratio $G^{c} / G_{o}^{c}$ of around 0.8 . This finding is consistent with a recent experimental investigation reported by (Ichii and Mikami 2018) where a cyclic torsional shear test was applied on clayey soils. When the stiffness of the soil drops to around $80 \%$ of its initial value, the excess pore water pressure begins to accelerate rapidly, thereby causing soil instability. In this case, a critical threshold for $G^{c}$ can be proposed, i.e., $80 \%$ of the initial $G^{c}$, and any 
further loss in $G^{c}$ than this level would cause soil to turn into an unstable stage where the EPWP and shear strain begins to increase rapidly. However, it is important to carry out further experimental investigations to examine how this critical threshold of $G^{c}$ can change with different soil and loading conditions.

Table 2 summarizes the values of shear modulus $G$ that are computed at different scales of shear strain, i.e., $G^{b}$ at $\varepsilon_{s}<10^{-5}$ by the BE and $G^{c}$ at $10^{-2}<\varepsilon_{s}<10^{-1}$ by cyclic triaxial (CTT) tests. Note that the values of $G^{c}$ at stable specimens (i.e., $C S R=0.2$ for 1620 and 1710 $\mathrm{kg} / \mathrm{m}^{3}$ density, and $C S R=0.3$ for $1805 \mathrm{~kg} / \mathrm{m}^{3}$ density) are considered in this analysis. The results show a significant change in $G$ at different levels of strain. For example, the value of $G^{c}$ for the specimen having a density of $1805 \mathrm{~kg} / \mathrm{m}^{3}$ is $60.2 \mathrm{MPa}$, while it is about $107.6 \mathrm{MPa}$ obtained through BE method. While the variation of $G$ over different strains has been widely reported in the literature (Sun et al. 1988; Shibuya et al. 1995; Kallioglou et al. 2008), specific values of shear modulus determined for subgrade soil collected from a real-time mud pumping site in this study is valuable for practicing engineers, especially considering different magnitudes of shear strain that the subgrade soil can experience under different railway loading conditions.

\section{Influence of kaolin content on the degradation of $G$}

Inclusion of kaolin has a considerable effect on the degradation of shear modulus of soil under cyclic loading. Fig. 7 shows that by adding $10 \%$ kaolin content, $G^{c}$ can better maintain itself compared to the original subgrade soil (K0), given the same shear strain. For example, while K0 specimen loses about $35 \%$ its shear strength at $\varepsilon_{s}=2 \%$, K10 specimen losses a smaller amount of $G^{c}$, i.e., $26 \%$ at the same level of $\varepsilon_{s}$. However, increasing $c_{K}$ to $30 \%$, on the other hand, makes $G^{c}$ decrease earlier and faster. In fact, $G^{c}$ reduces by $35 \%$ for the K30 specimen at $\varepsilon_{s}=0.5 \%$, whereas $\mathrm{K} 0$ and $\mathrm{K} 10$ remain at about $90 \%$ of their initial shear 
strength at the same degree of shear strain. This is consistent with previous findings (Indraratna et al. 2020a) where the inclusion of $10 \%$ kaolin was shown to be effective in mitigating early softening and associated fluidization of the subgrade soil (i.e., K0 specimen). Mamou et al. (2017a) also showed increasing clay content can help enhance the cyclic shear stress threshold and reduce the build-up EPWP. Despite the different kaolin contents, Fig. 7 reaffirms that the soil can experience a swift increase in EPWP and shear strain (i.e., the critical number of cycles $N_{c}$ ) when $G^{c} / G_{o}^{c}$ becomes smaller than 0.8 (highlighted by red dots in Fig. 7).

\subsection{Damping properties of subgrade soil}

It is agreed upon that the behaviour of damping ratio $\left(D_{p}\right)$ is governed by the same factors as the shear modulus $G$ such as shear strain, OCR, PI, initial effective stress and void ratio (or density); albeit in an opposite trend to that of $G$, i.e., the reduction in $G$ occurs in conjunction with the increase in $D_{p}$ over increasing $\varepsilon_{s}$ (Sun et al. 1988; Vucetic and Dobry 1991; Rollins et al. 1998; Kallioglou et al. 2008). Indeed, the degradation in shear modulus also means the degradation in the soil fabric and internal contact network of the soil grains, resulting in soil fluidization (Nguyen and Indraratna 2020a). This, in turn, results in increased energy dissipation while transmitting the cyclic load through the soil skeleton, leading to a larger damping ratio $D_{p}$. As there is a lack of previous studies addressing the effect of CSR and loading cycles on $D_{p}$, the following section is aimed to clarify this issue in light of varying kaolin content and soil density.

\section{Influence of CSR and soil density}

The influence of CSR and soil density on the damping characteristics of subgrade soil are considered in tandem in this section. Fig. 8 represents the values of $D_{p}$ measured for specimens at different densities with varying CSR. It is apparent that there are two distinct 
trends in the behaviour $D_{p}$, they are: (i) $D_{p}<0.1$ in stable specimens and (ii) $D_{p}>0.15$ in unstable specimens. Particularly when $C S R<C S R_{c}$, the specimen is stable at small scale of strain, i.e., $<0.1 \%$ up to 1,000 cycles, resulting in a small value of $D_{p}$ (i.e., $D_{p} \leq 0.1$ ). However, when $C S R>C S R_{c}$, for example, at $C S R \geq 0.3$ for the soil specimen compacted at a density of $1620 \mathrm{~kg} / \mathrm{m}^{3}, D_{p}$ can exceed 0.17 and reach 0.22 at $C S R=0.5$. This is because at a critical level of $C S R$, the soil experiences a considerable high magnitude of shear strain even at its initial loading cycles, causing a large amount of cyclic energy being trapped through the strain evolution. Fig. 8d showcases the degradation in the hysteresis loops for an unstable soil subjected to $C S R>C S R_{c}$. As larger energy is being stored with each loading cycle, the specimen would tend to fail in fewer loading cycles. A rapid increase in $D_{p}$ is observed for all unstable specimens as $N$ increases, and this corresponds to large accumulated plastic strains in the soil specimen.

To propose an objective view into the effects of soil density and $C S R$, the average damping ratios at stable periods, i.e., (i) stable specimens where $C S R<C S R_{c}$, and (ii) unstable specimens where $C S R \geq C S R_{c}$ but $N<N_{c}$ (before failure) are represented over different values of dry density and void ratio (Fig. 9). The results indicate a sudden decrease in $D_{p}$ as $\rho_{d}$ increases from 1710 to $1805 \mathrm{~kg} / \mathrm{m}^{3}$; for example, $D_{p}$ reduces from 0.21 to 0.075 at $\mathrm{CSR}=0.5$, however $D_{p}$ does not change significantly as $\rho_{d}$ increases from 1620 to 1710 $\mathrm{kg} / \mathrm{m}^{3}$. This clearly indicates a certain threshold value of soil density is required to cause a significant change in $D_{p}$. This threshold of soil density can be determined more accurately by carrying out tests at different soil densities, for example, within the range $1710-1805 \mathrm{~kg} / \mathrm{m}^{3}$ at $\mathrm{CSR}=0.5$, however, this would warrant further research due to its innate complexity. The denser the soil particles, the stiffer the soil specimen, and hence lesser the energy induced by the cyclic excitation which can be absorbed into the soil. 


\section{Influence of kaolin content on damping properties}

Figure 10 shows that the addition of kaolin can affect the value of $D_{p}$ significantly. For example, for about 20 initial cycles, $D_{p}$ drops from about 0.18 to nearly 0.09 for $N<20$ cycles, when $10 \%$ and $30 \%$ kaolin contents are added to the original soil. This indicates that less cyclic energy is being transferred to the kaolin-added specimens, which explains why there is less build-up of excess pore pressure in these kaolin-added specimens compared to the original soil. It is important to revisit the findings from a previous study (Indraratna et al. 2020a) in which soil added with $10 \%$ and $30 \%$ kaolin contents exhibited a smaller increase in excess pore water pressure, and thus lesser internal re-distribution of water content. However, it is interesting to note that $10 \%$ kaolin helps to stabilize the value of $D_{p}$ better; for example, the $D_{p}$ of K30 specimen begins to increase rapidly when $N$ approaches the critical level of about 80 (also see Indraratna et al. (2020a), whereas the $D_{p}$ of K10 specimen rises at a slower rate over the same number of cycles. Fig. 10b shows different responses of K0, K10 and K30 specimens with increasing shear strain $\varepsilon_{\mathrm{s}}$. While the $D_{p}$ of $\mathrm{K} 0$ and $\mathrm{K} 30$ specimens begins to increase rapidly when $\mathcal{E}_{s}>0.2 \%$, the $D_{p}$ of K10 specimen still increases but at a reduced rate for the same value of $\varepsilon_{s}$. This indicates the performance of $10 \%$ kaolin in maintaining the value of $D_{p}$. Despite these differences, it is believed that all soil specimens experience a considerable change in $D_{p}$ when $\mathcal{E}_{s}$ exceeds $0.4 \%$.

The range of $D_{p}$ (i.e., 0.05 to 0.1 in stable specimens $C S R<C S R_{c}$ and $\varepsilon_{s}<0.1 \%$ ) obtained for the subgrade soil used in the current study generally agrees with previous studies (Vucetic et al. 1998a) where $D_{p}$ of different specimens was found to vary from 0.04 to 0.14 considering the same degree of shear strain. In comparison to the overlying ballast in which a higher loading frequency (e.g., 10 to $40 \mathrm{~Hz}$ ) is normally considered, the value of $D_{p}$ seems to be smaller. Specifically, the $D_{p}$ of ballast can vary over a wider range; for example, from 0.05 up to 0.3 considering the same level of shear strain (Esmaeili et al. 2016; Navaratnarajah and 
Indraratna 2017). This suggests that ballast can dissipate kinetic energy better than a clayey subgrade attributed to its much higher internal friction, which also explains why dynamic stress and frequency usually attenuate considerably more through the ballast layer (Priest et al. 2010).

\section{Empirical model to determine the degradation of shear modulus at high CSR}

\subsection{Conceptual rationale and model development}

The use of empirical models to estimate the degradation in soil shear strength is often preferred in practice due to its simplicity. However, earlier models have relied on the development of shear strain (Vucetic and Dobry 1991; Kallioglou et al. 2008) while the role of loading parameters has been less understood properly. In essence, the loading parameters such as $C S R$ and $N$ are usually the first input that can practically be estimated while assessing the dynamic response of a rail track, so they are adopted herein for determining the degradation of the subgrade soil. Furthermore, it is important to note that while PI can influence significantly the degradation of shear modulus, it is not considered in this model because the current study focuses on low to medium plasticity soils (PI $=11-15$ for the adopted experimental data) which are susceptible to pumping under rail tracks.

As shown in the above sections, for a given subgrade soil, the degradation ratio $G^{c} / G_{o}^{c}$ primarily depends on $C S R$ and $N$ while the frequency is kept unchanged in this study, i.e. $f=5 \mathrm{~Hz}$. This relationship can be generalized as follows:

$$
G^{c} / G_{o}^{c}=1-f(C S R) g(N)
$$

where $f(C S R)$ and $g(N)$ are the mathematical functions representing the influence of $C S R$ and $N$, respectively. As the ratio $G^{c} / G_{o}^{c}$ does not change significantly when $C S R<C S R_{c}$ despite 
$N$ increasing (stable specimens), the current study addresses substantial deterioration which occurs when $C S R>C S R_{c}$ (i.e., $G^{c} / G_{o}^{c}$ exceeds the critical ratio 0.8 accompanied with rapid increase in shear strain and excess pore pressure). It can also be observed from Figs. 5 and 6 that there are 2 distinctly different forms that the degradation plot can represent, i.e., $(i)$ gradual degradation during initial stages followed by a swift reduction in $G$ (undrained cyclic failure); and (ii) early degradation following a short period of stability during the initial stage (early softening failure) (see Fig. 11).

For the soil to be unstable under cyclic loading, the power function is usually used to represent the effect of $N$ on the accumulated strain and pore water pressure (Li and Selig 1996; Konstadinou and Georgiannou 2014), while there are only limited empirical models (Cai et al. 2018) that can capture the influence of CSR on early softening. The current study also proposes a power function to simulate the effect of increasing $N$ whereas, the logarithmic function of $N$ is found to incorporate the function of CSR more accurately. Specifically, the empirical equation to estimate soil degradation can now be represented as follows:

$$
G^{c} / G_{o}^{c}=1-\log (a \times C S R)^{b}[\log (N)]^{c}
$$

where $a$ and $b$ are the parameters governing the effect of $C S R$, while $c$ is used to evaluate the influence of $N$. In essence, these parameters change with different soil properties such as the density (void ratio) and the associated failure modes of the soil specimens.

\subsection{Model applications and discussion}

Table 3 summarizes parameters $a, b$ and $c$ which can be used to estimate the degradation ratio $G^{c} / G_{o}^{c}$ of the subgrade soil specimens used in the current study. It is interesting that $a$ is quite similar (i.e., $a=3.2$ and 3.34) for the specimens of density 1620 and $1710 \mathrm{~kg} / \mathrm{m}^{3}$, which can be attributed to the fact that these specimens fail within the same range of CSR (i.e., 0.3-0.5). For the higher density specimen i.e.., $\rho_{d}=1805 \mathrm{~kg} / \mathrm{m}^{3}$ where the critical value $C S R_{c}$ causing 
the soil to soften early increases substantially to 1.0 , the parameter $a$ increases to 4.6 . Especially when considering the same soil (i.e., the density $1710 \mathrm{~kg} / \mathrm{m}^{3}$ ) but different failure modes, the value of $a$ is unchanged while the parameter $c$ representing the influence of $N$ jumps from 2.5 to 7.1. This variation in the values of $a, b$ and $c$ reasonably reflects that a larger number of cycles is usually needed to cause undrained cyclic failure compared to early softening, where an apparently high degree of CSR can induce the soil to fail quickly during the initial loading cycles. In addition, an application of Eq. [6] to other independent studies (Głuchowski and Sas 2020) where the degradation in shear strength of a similar soil (PI = 12.5 at shallow subgrade under road) over the number of cycles is captured is carried out. Table 3 shows that parameter $a$ increases to 6.7 in this case due to a larger dry density $\left(\rho_{d}=\right.$ $1935 \mathrm{~kg} / \mathrm{m}^{3}$ ) while $c$ becomes smaller because of the less impact from $N$ (i.e., the larger $\mathrm{N}$ needed to cause soil degradation at small level of CSR).

Fig. 12 shows the estimated results in comparison with the experimental data. While a good agreement is found with the experimental data for undrained failure, the estimated vales for $G^{c} / G_{o}^{c}$ match quite well with the experimental data for the early softening cases, albeit certain deviations at some values of $N$. The mean absolute percentage errors which represent the difference between the estimated and experimental data are within acceptable margins, i.e. about $3.0 \%$ and $4.5 \%$ for the undrained and early softening failures, respectively. Fig. 12c also indicates an excellent agreement between the proposed model and experimental data for slow degradation cases ( $N$ up to 15,000 cycles). Although empirical methods such as this cannot always explain insightfully the mechanisms of soil degradation, they can still bring considerable practical value due to its simplicity and relevant outcomes.

Fig. 13 represents a 3D plot showing the variation of shear modulus degradation with the loading parameters (i.e., $C S R$ and $N$ ) on the basis of the proposed empirical relationship. In this illustration, two cases of soil density 1620 and $1710 \mathrm{~kg} / \mathrm{m}^{3}$ are used for comparison as 
both of these soil specimens experience early softening in the similar range of CSR (i.e., > 0.3). The $3 \mathrm{D}$ surface shows the drop in the degradation ratio $G^{c} / G_{o}^{c}$ with increasing $C S R$ and $N$. For example, increasing the value of $C S R$ requires a smaller number of cycles $N$ to cause the same degree of degradation. The larger the specimen density, the larger the resistance to cyclic instability as reflected by a reduced slope in the plot (Fig. 13). Although the current study was able to capture the possible shape of early softening surface when a subgrade soil is subjected to an increasing cyclic loading, further experimental data is still needed to enhance the model validity especially considering different soils. Moreover, as the current model is mainly based on CSR, more effort is needed to incorporate the effect of soil parameters such as the soil density or void ratio on the dynamic response of soils.

It is also worth noting that although an empirical model to determine damping ratio is not addressed in this paper, $D_{p}$ can be back-calculated based on $G^{c} / G_{o}^{c}$ given by Eq. [6]. For example, for a very narrow range of PI, i.e., 11-15 considered in this study, $D_{p}$ can be obtained through a polynomial relationship with $G^{c} / G_{o}^{c}$ as suggested by Park and Stewart (2001). Because the relationship between $D_{p}$ and $G^{c} / G_{o}^{c}$ has been addressed extensively in past studies (Vucetic and Dobry 1991; Park and Stewart 2001; Kallioglou et al. 2008), the current paper does not further attempt to establish an empirical model for $D_{p}$.

\section{Conclusions}

This paper represented an analysis of dynamic parameters including the shear wave velocity $\left(V_{s}\right)$, the shear modulus $(G)$ and the damping ratio $\left(D_{p}\right)$ of subgrade soil considering varying kaolin content $\left(c_{K}\right)$ and loading parameters under heavy haul rail tracks. Bender element (BE) test was used to examine $V_{s}$ while the cyclic triaxial test (CTT) results were used to estimate $G$ and $D_{p}$. The salient findings of this study lead to the following conclusions.

Increasing the kaolin content resulted in a decreased value of $D_{50}$, thus reducing the 
value of $V_{s}$, e.g., $V_{s}$ decreased from $184 \mathrm{~m} / \mathrm{s}$ to $162 \mathrm{~m} / \mathrm{s}$ when $c_{K}$ increased from 0 to $30 \%$. The study also indicated a certain threshold of void ratio, $e$ (e.g. 0.85-0.9) where soils with smaller value of $e$ would result in a swift increase in the magnitude of $V_{s}$. The value of shear modulus based on $V_{s}$ (i.e., $G^{b}$ at small strain) was found at about 107.6 MPa which was 1.7 times greater than the largest value of $G^{c}$ (i.e. $60.2 \mathrm{MPa}$ ) based on CTT results for the same soil density of $1805 \mathrm{~kg} / \mathrm{m}^{3}$.

When CSR exceeded a critical level $\mathrm{CSR}_{\mathrm{c}}, G^{c}$ decreased apparently at early stage, for example, it lost about $10 \%$ during initial 30 cycles for a density of $1620 \mathrm{~kg} / \mathrm{m}^{3}$ and CSR of 0.4. When the degradation index $G^{c} / G_{o}^{c}$ exceeded a critical level of about 0.8 , the soil specimen began to experience a rapid increase in excess pore pressure and strain. The study also indicated considerable influence kaolin content on the ratio of $G^{c} / G_{o}^{c}$. For example, $10 \%$ addition of kaolin helped mitigate the degradation rate of $G^{c}$ significantly, whereas $30 \%$ kaolin worsened the degradation rate. An empirical equation to estimate $G^{c} / G_{o}^{c}$ for the current medium plasticity soil under cyclic loading was proposed with acceptable accuracy for capturing early softening behaviour of soil at high levels of CSR.

Adding kaolin into the current moderately plastic soil reduced the damping ratio $D_{p}$ significantly, for example, from 0.18 to 0.09 when $10 \%$ kaolin was included. Moreover, $10 \%$ kaolin was found to better stabilize $D_{p}$ over increasing $N$. The damping ratio $D_{p}$ increased significantly when $\mathrm{CSR} \geq \mathrm{CSR}_{\mathrm{c}}$ where the cyclic resistance substantially deteriorated. Also at these high values of CSR, $D_{p}$ could exceed 0.2 at low to medium dry density (1620 and 1710 $\left.\mathrm{kg} / \mathrm{m}^{3}\right)$ and reach 0.15 at a well-compacted state $\left(1805 \mathrm{~kg} / \mathrm{m}^{3}\right)$. However, for CSR $<\mathrm{CSR}_{\mathrm{c}}, D_{p}$ was found to be less than 0.1 . 


\section{CRediT author statements}

Thanh Trung Nguyen: Conceptualization, Writing-Original Draft, Methodology, Formal analysis, Resources; Buddhima Indraratna: Writing-Review \& Editing, Funding Acquisition, Resources; Mandeep Singh: Writing-Review, Editing and Analysis

\section{Acknowledgements}

This research was supported by the Australian Government through the Australian Research Council's Linkage Projects funding scheme (project LP160101254) and the Transport Research Centre (TRC), University of Technology Sydney. The financial and technical supports from SMEC, Coffey, ARTC (Australian Rail Track Corporation) and Sydney Trains are acknowledged. The data and supports from by Dr. Warrantorn Korkitsuntornsan are much appreciated. 


\section{Tables}

Table 1 Variation of soil properties with kaoline content $\left(c_{K}\right)$

\begin{tabular}{|c|c|c|c|c|}
\hline \multirow{2}{*}{ Soil parameters } & \multicolumn{3}{|c|}{$\begin{array}{l}\text { Subgrade soil mixed with } \\
\qquad \text { kaolin }\end{array}$} & \multirow{2}{*}{$\begin{array}{c}\text { Original } \\
\text { kaolin }\end{array}$} \\
\hline & $\begin{array}{c}c_{K}=0 \% \\
\text { (original soil) }\end{array}$ & $c_{K}=10 \%$ & $c_{K}=30 \%$ & \\
\hline Percentage of clay $(<2 \mu \mathrm{m}), \%$ & 9.5 & 10.1 & 12 & 58 \\
\hline Percentage of silt $(2-75 \mu \mathrm{m}), \%$ & 45.3 & 52.4 & 58.2 & 42 \\
\hline Percentage of sand $(>75 \mu \mathrm{m}), \%$ & 45.2 & 37.5 & 29.8 & 0 \\
\hline$D_{50}$ & 0.05 & 0.022 & 0.013 & 0.01 \\
\hline Specific gravity, $\mathrm{G}_{\mathrm{s}}$ & 2.65 & 2.63 & 2.62 & 2.61 \\
\hline Liquid limit, LL (\%) & 26 & 28 & 31 & 51 \\
\hline Plastic limit, PL (\%) & 15 & 15 & 16 & 29 \\
\hline Plastic index, PI (\%) & 11 & 13 & 15 & 22 \\
\hline
\end{tabular}


Table 2 Shear modulus of subgrade soil $\mathrm{K} 0$ determined by different methods

\begin{tabular}{|c|c|c|c|}
\hline \multirow{2}{*}{$\begin{array}{c}\text { Shear modulus } G \text { at } \\
\text { different levels of shear strain } \\
(\mathrm{MPa})\end{array}$} & \multicolumn{3}{|c|}{$\begin{array}{l}\text { Dry density } \\
(\text { void ratio } e)\end{array}$} \\
\hline & $\begin{array}{c}1620\left(\mathrm{~kg} / \mathrm{m}^{3}\right) \\
(e=0.64) \\
\mathrm{CSR}=0.2\end{array}$ & $\begin{array}{c}1710\left(\mathrm{~kg} / \mathrm{m}^{3}\right) \\
(e=0.55) \\
\mathrm{CSR}=0.2\end{array}$ & $\begin{array}{c}1805\left(\mathrm{~kg} / \mathrm{m}^{3}\right) \\
(e=0.47) \\
\mathrm{CSR}=0.3\end{array}$ \\
\hline $\begin{array}{l}G^{b} \text { by Bender element }(\mathrm{BE}) \text { test } \\
\left(\varepsilon_{s}<10^{-4}\right)\end{array}$ & 68.1 & 85.9 & 107.6 \\
\hline $\begin{array}{l}G^{c} \text { by Cyclic triaxial }(\mathrm{CT}) \text { tests } \\
\left(10^{-2}<\varepsilon_{s}<10^{-1}, \text { stable K0 specimens }\right)\end{array}$ & 39.8 & 48.4 & 60.2 \\
\hline
\end{tabular}


Table 3 Parameters for the proposed emprical model

\begin{tabular}{|l|c|c|c|}
\hline \multicolumn{1}{|c|}{ Soil density and failure modes } & \multicolumn{2}{|c|}{ Model parameters } \\
\cline { 2 - 4 } & $\mathrm{a}$ & $\mathrm{b}$ & $\mathrm{c}$ \\
\hline K0, $\rho_{d}=1620\left(\mathrm{~kg} / \mathrm{m}^{3}\right)$, & 3.2 & 2.1 & 1.6 \\
Early softening, & 3.34 & 2.0 & 7.1 \\
\hline $\begin{array}{l}\text { K0, } \rho_{d}=1710\left(\mathrm{~kg} / \mathrm{m}^{3}\right), \\
\text { Undrained cyclic failure }\end{array}$ & 3.34 & 0.69 & 2.5 \\
\hline $\begin{array}{l}\text { K0, } \rho_{d}=1710\left(\mathrm{~kg} / \mathrm{m}^{3}\right), \\
\text { Early softening; }\end{array}$ & 4.6 & 0.3 & 3.1 \\
\hline $\begin{array}{l}\text { K0, } \rho_{d}=1805\left(\mathrm{~kg} / \mathrm{m}^{3}\right), \\
\text { Early softening }\end{array}$ & 6.7 & 1.8 & 1.1 \\
\hline $\begin{array}{l}\text { Subgrade soil used by }(\mathrm{Gluch} \\
\text { Sas 2020), } \rho_{d}=1935\left(\mathrm{~kg} / \mathrm{m}^{3}\right) \\
\text { Slow degradation }\end{array}$ & & \\
\hline
\end{tabular}




\section{Figures}

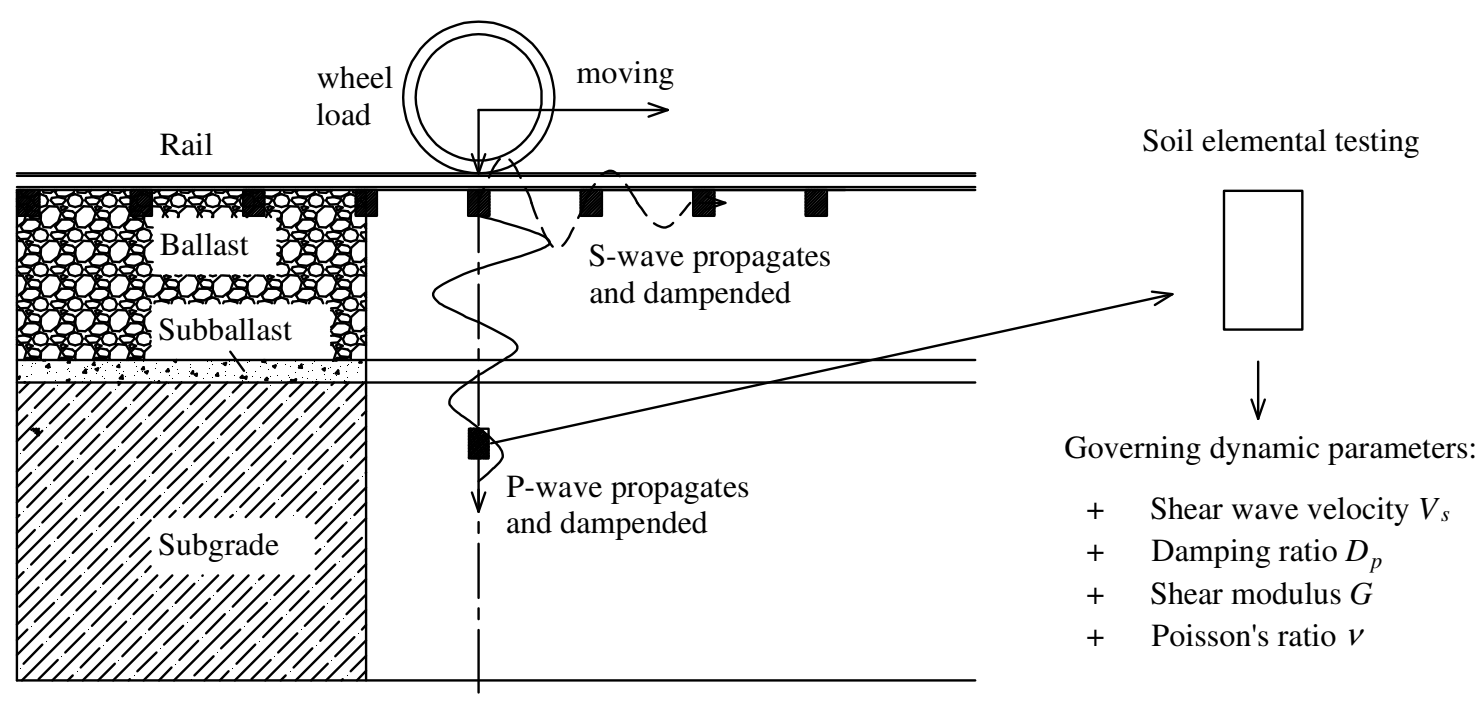

Fig. 1 Dynamic parameters of railway foundation determined through laboratory testing scheme 


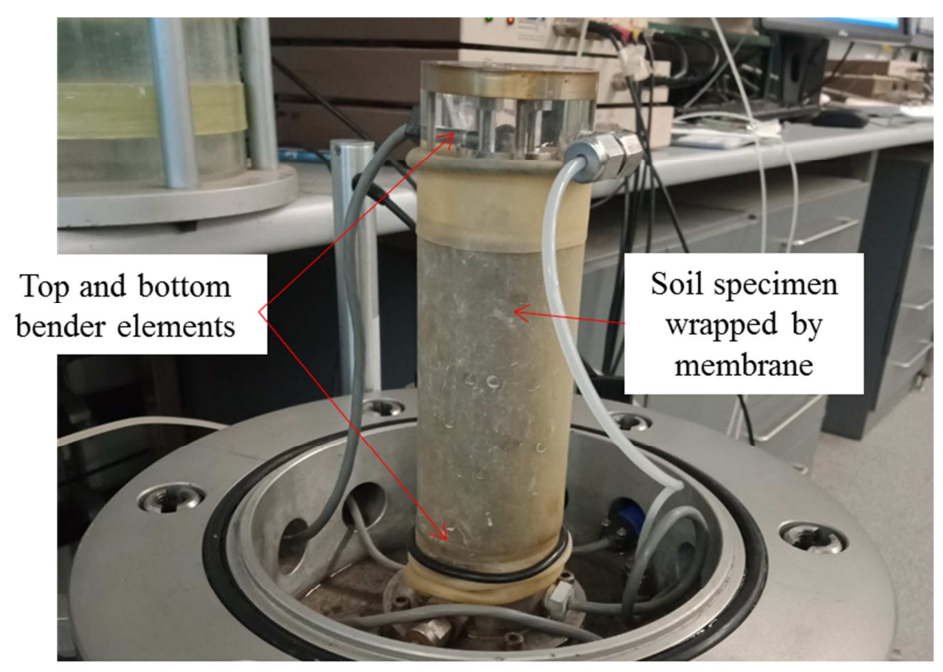

Specimen size: $50 \times 100 \mathrm{~mm}$

a)

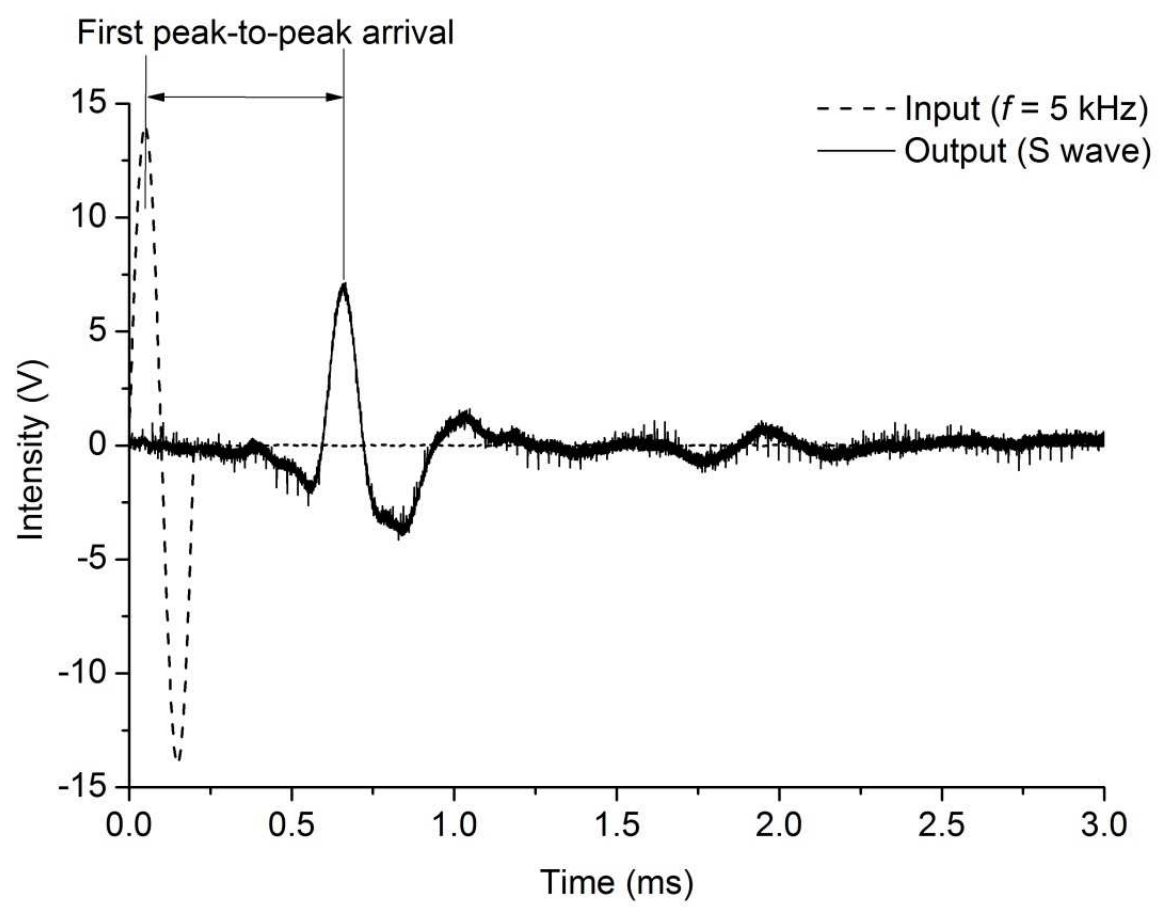

b)

Fig. 2 Bender element testing on specimens: a) installation of Bender elements; and b) representative shear (S) wave transmitting through the specimen $\left(\mathrm{K} 30, \rho_{d}=1620 \mathrm{~kg} / \mathrm{m}^{3}\right)$ 


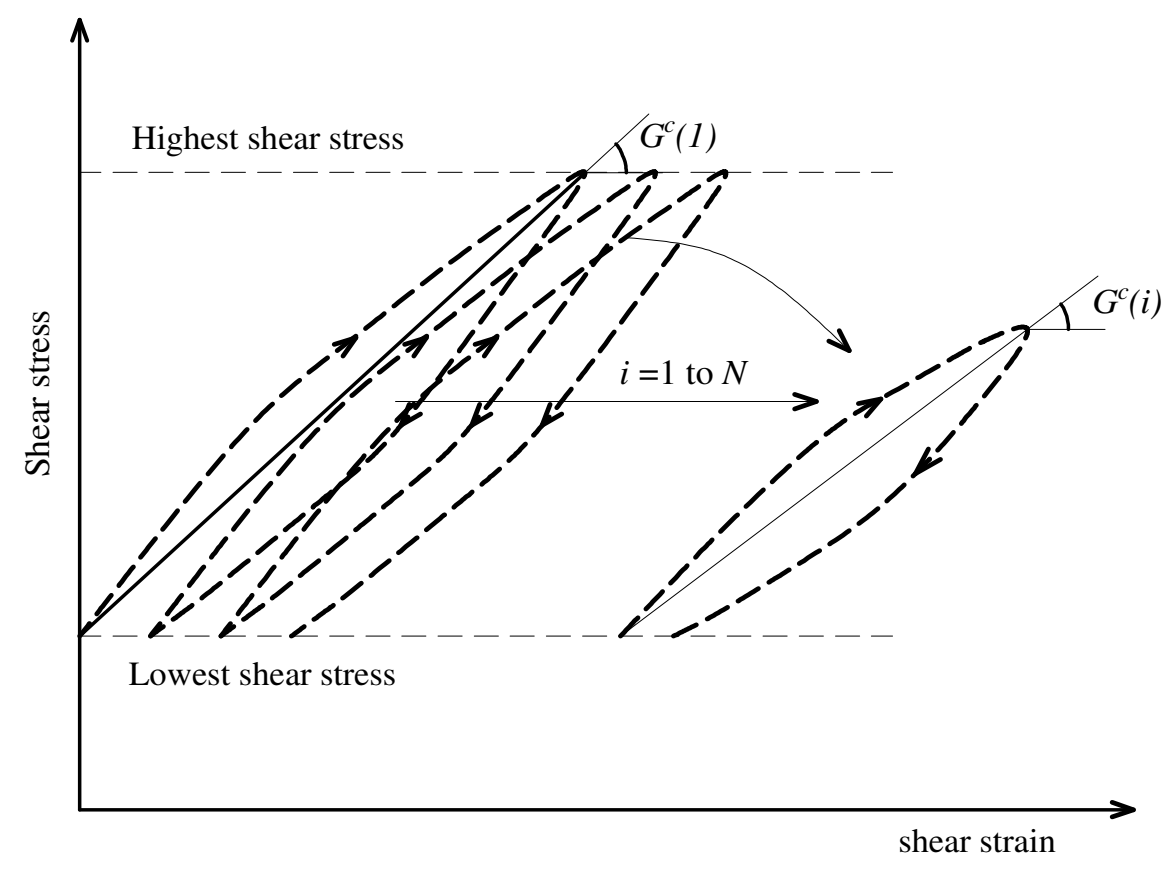

a)

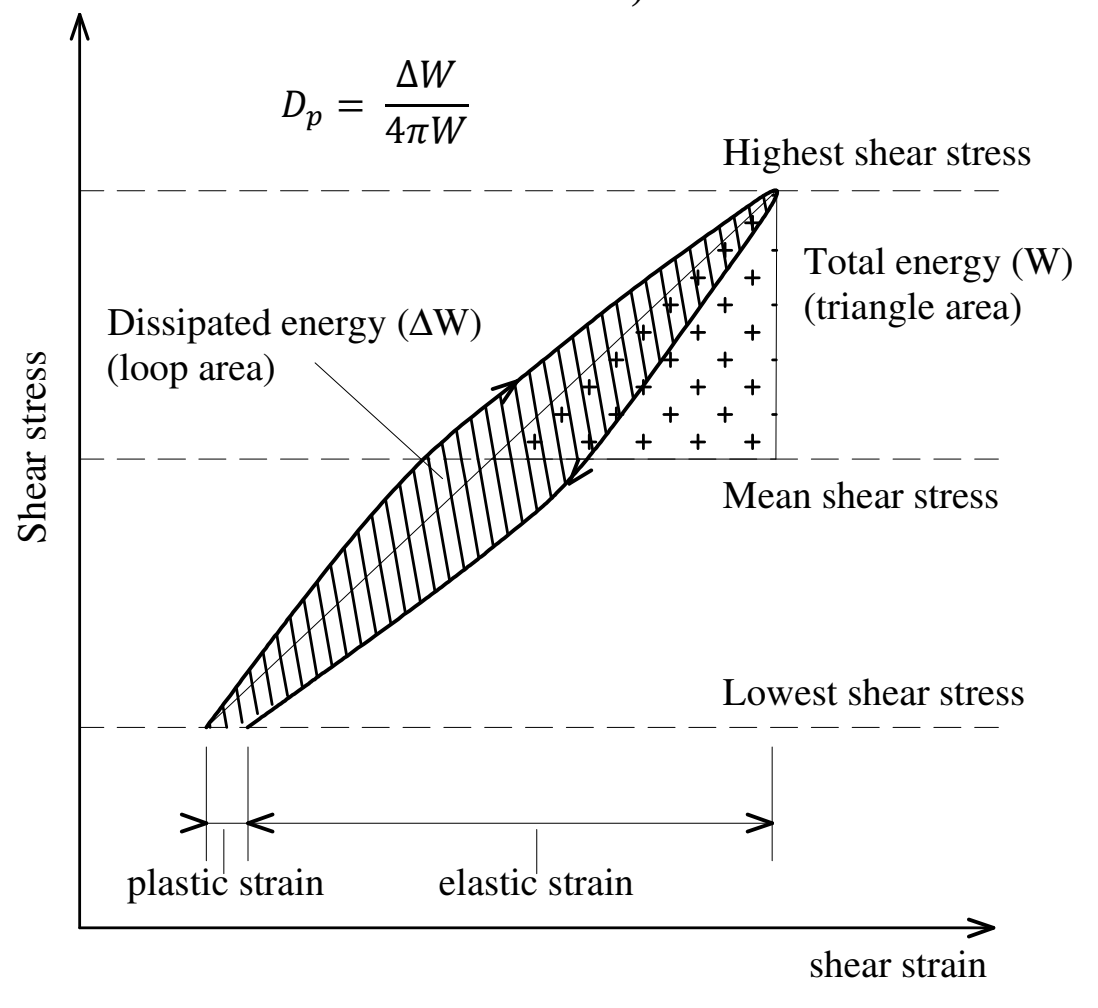

b)

Fig. 3 Theoretical concepts to estimate dynamic parameters based on cyclic triaxial test data: a) shear modulus $G^{c}$; b) damping ratio $D_{p}$ 


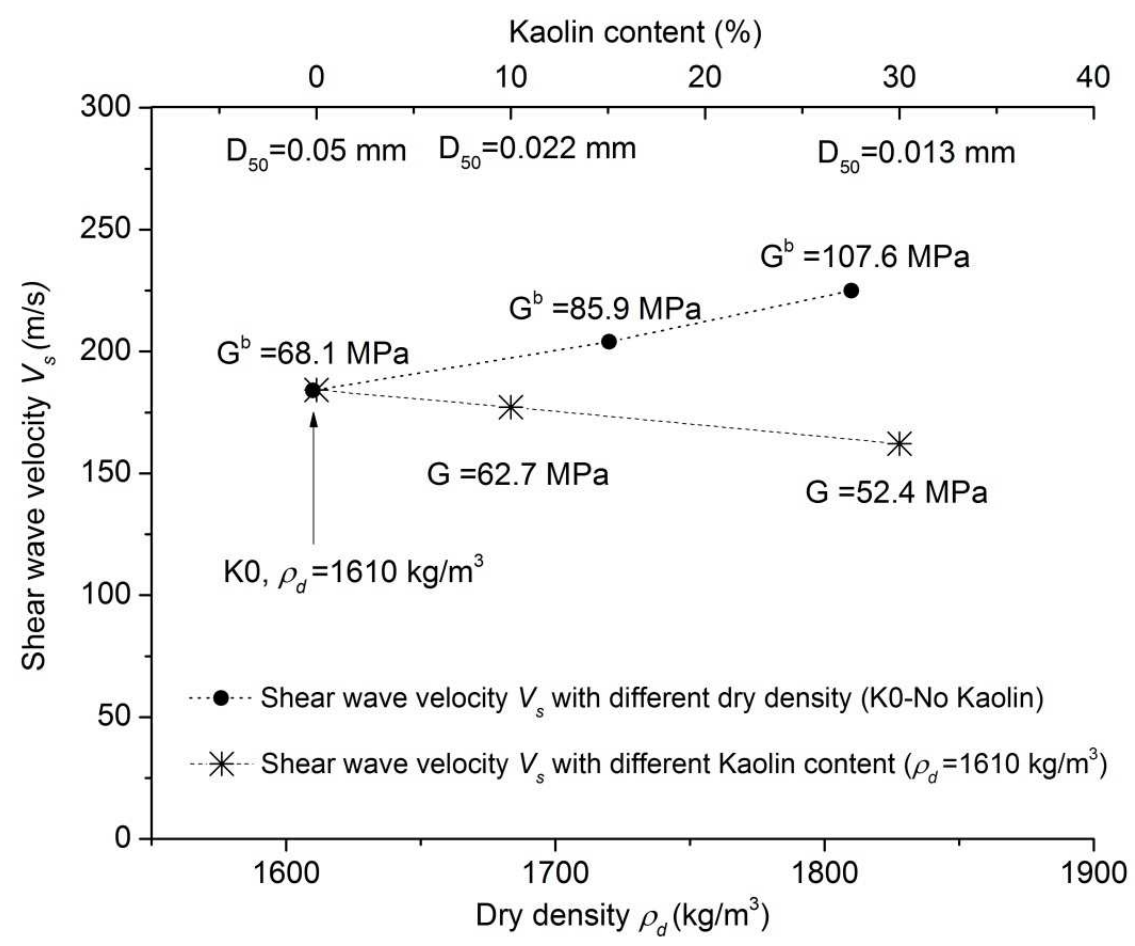

a)

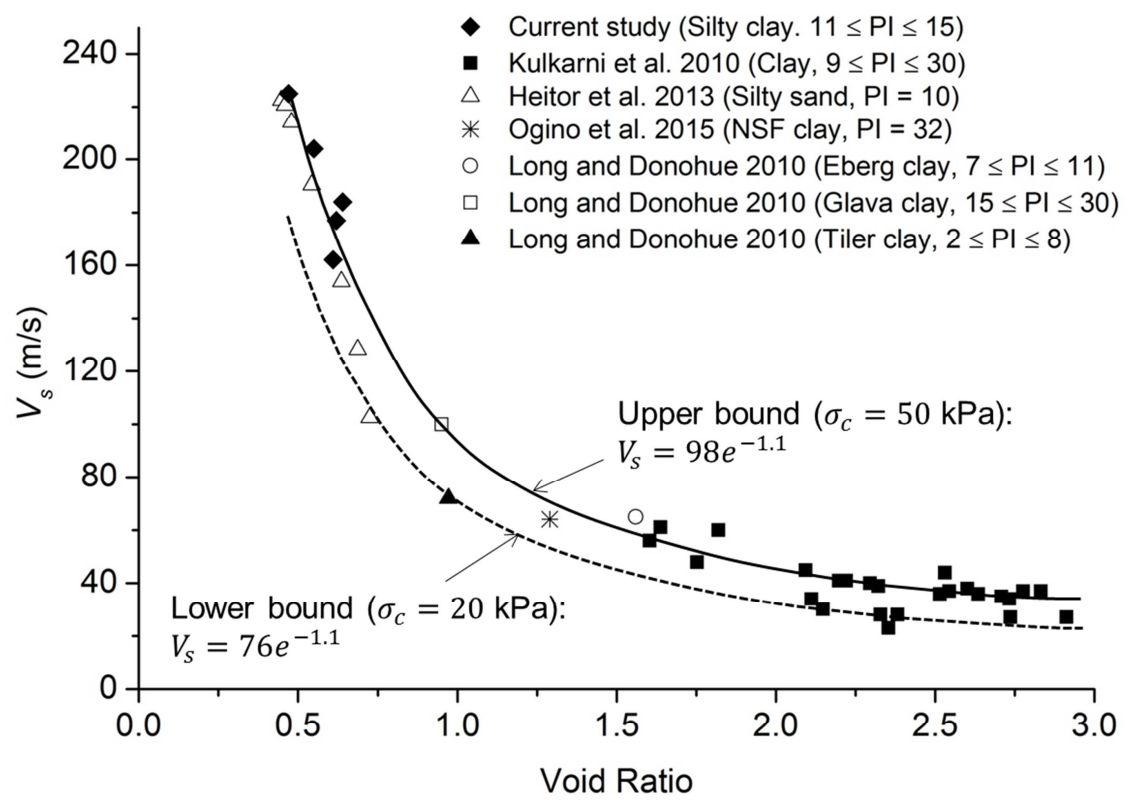

b)

Fig. 4 Variation of shear wave velocity with: a) different dry densities and kaolin contents (current study); and b) void ratio in comparison with previous studies 


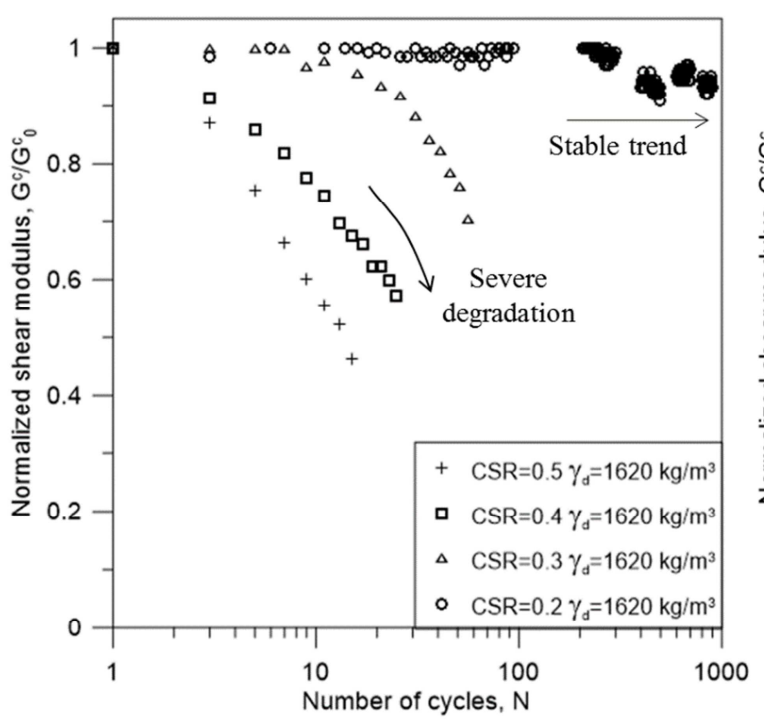

a)

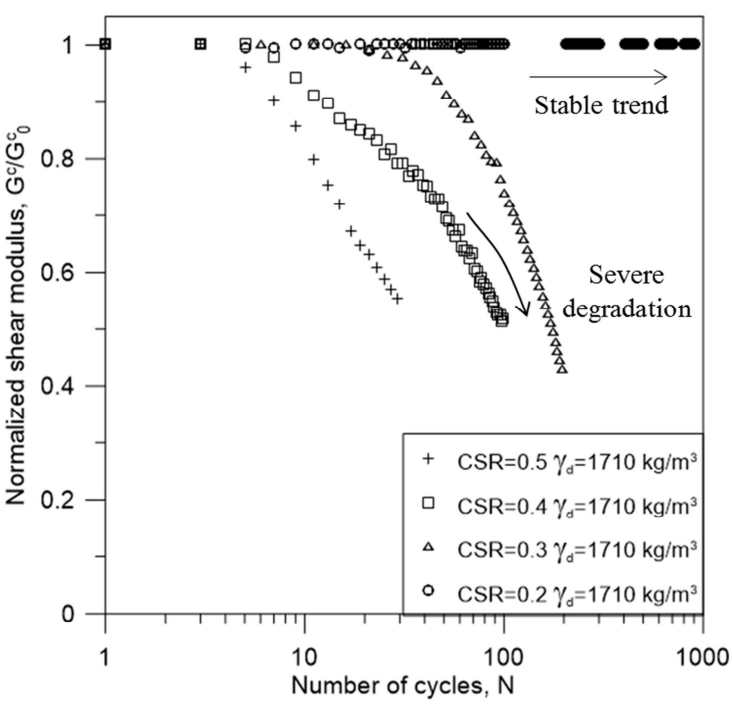

b)

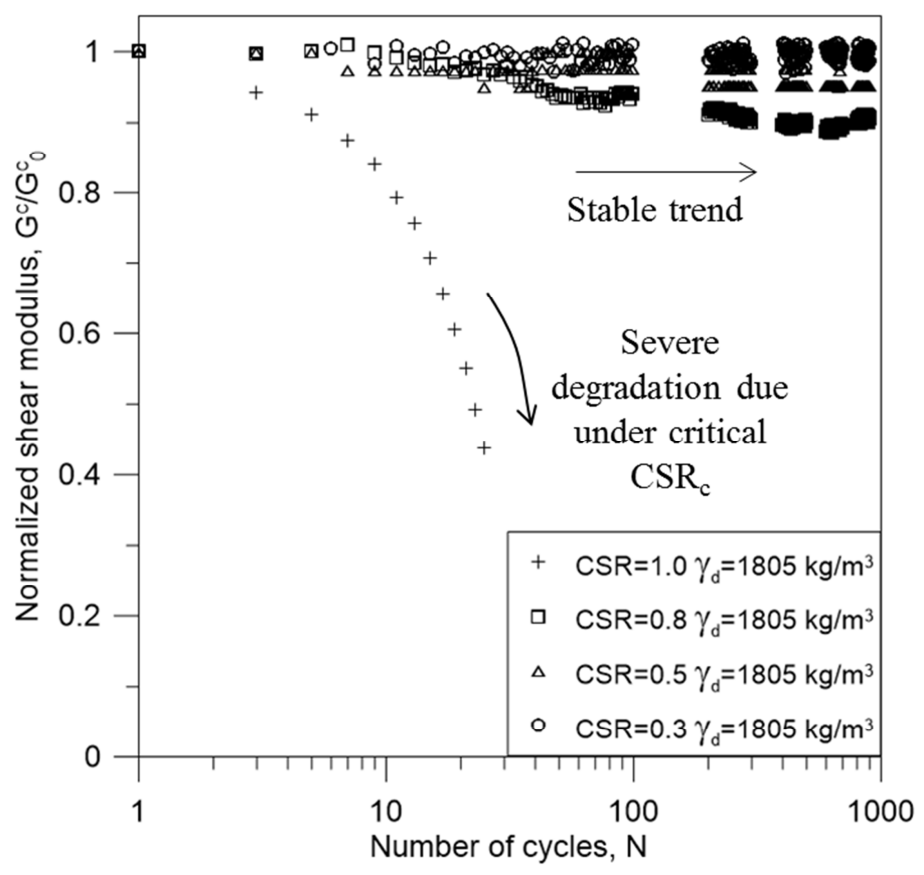

c)

Fig. 5 Influence of CSR on the degradation of $G^{c}$ while $N$ increases: a) $\rho_{d}=1620 \mathrm{~kg} / \mathrm{m} 3$; b) $\left.\rho_{d}=1710 \mathrm{~kg} / \mathrm{m} 3 ; \mathrm{c}\right) \rho_{d}=1805 \mathrm{~kg} / \mathrm{m} 3$ 


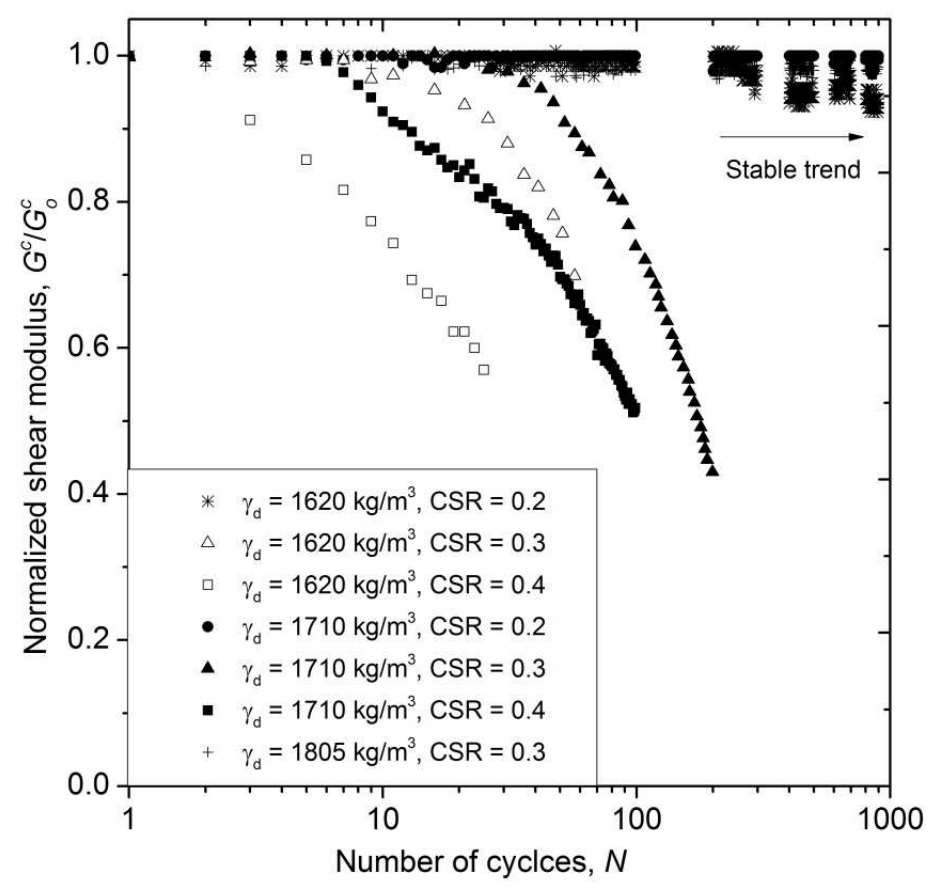

a)

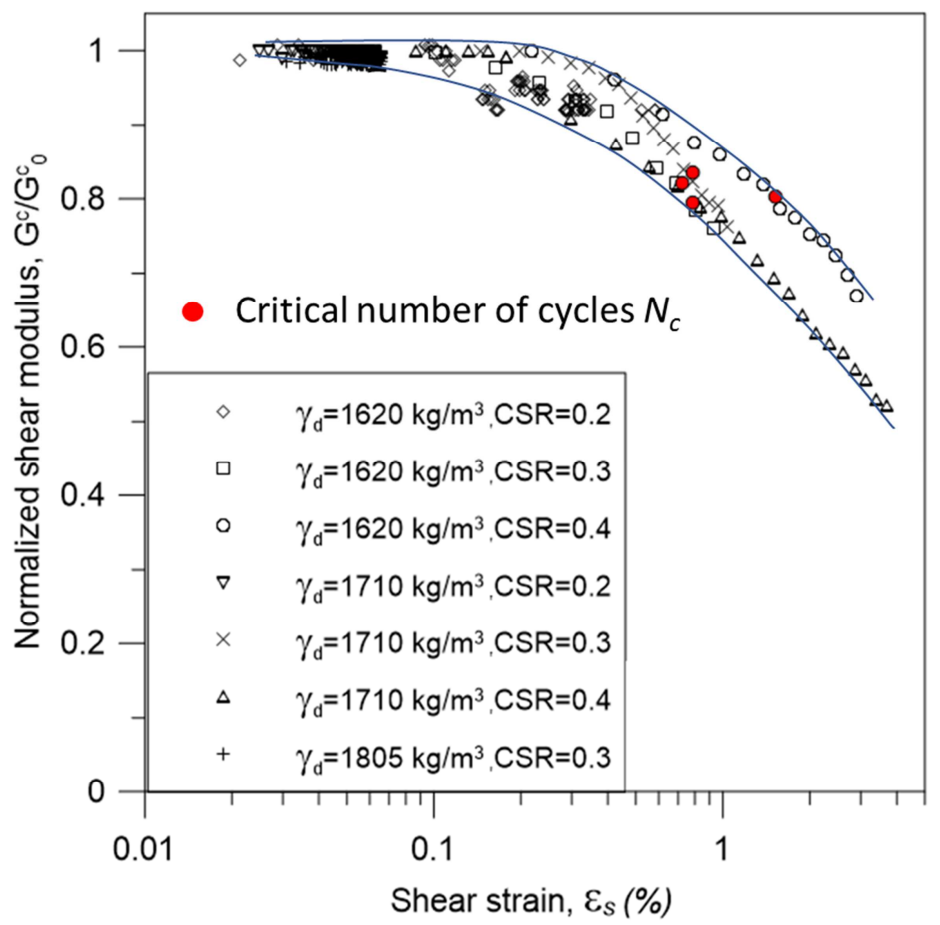

b)

Fig. 6 Influence of soil density (or void ratio) on the degradation of $G^{c}$ a) with number of cycles $N$; and b) with shear strain $\mathcal{E}_{s}$ 


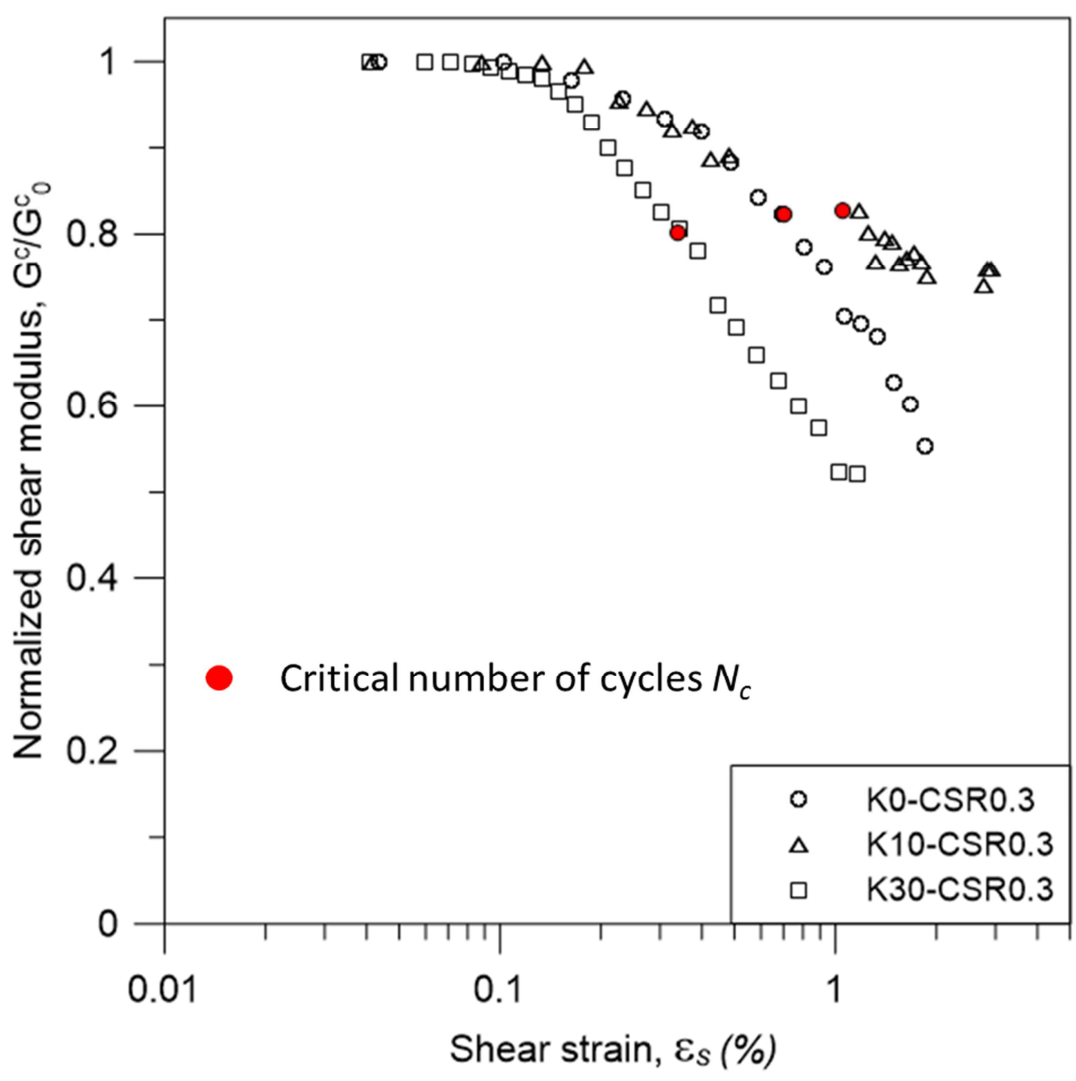

Fig. 7 Effects of kaolin content on the degradation of $G^{c}$ at critical value of CSR (0.3) 


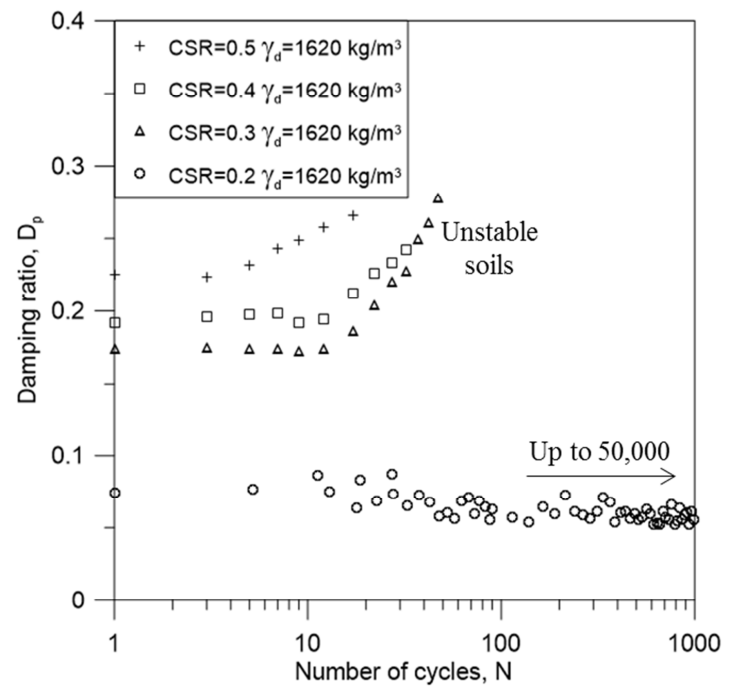

a)

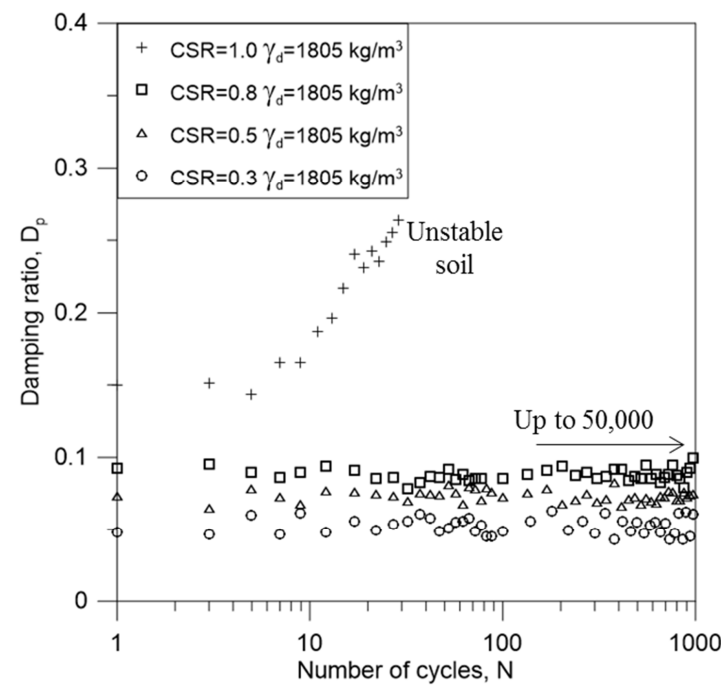

c)

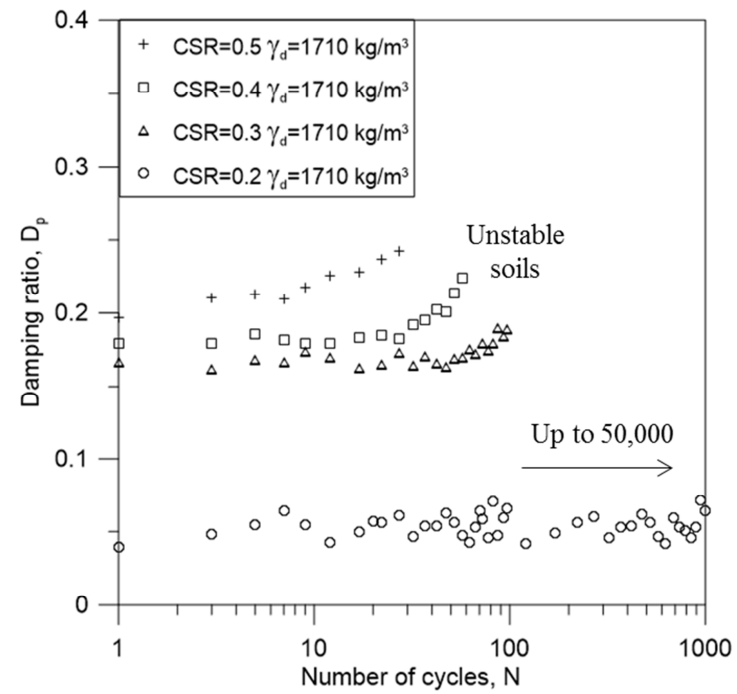

b)

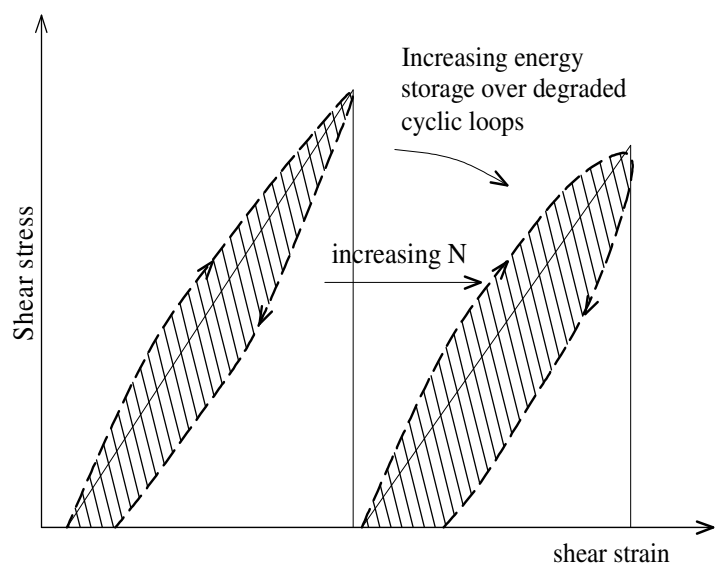

d)

Fig. 8 Damping ratio changes with varying CSR over different densities: a) $\rho_{d}=1620 \mathrm{~kg} / \mathrm{m}^{3}$;

b) $\rho_{d}=1710 \mathrm{~kg} / \mathrm{m}^{3}$; c) $\rho_{d}=1805 \mathrm{~kg} / \mathrm{m}^{3}$, and d) mechanism of increasing $D_{p}$ over degraded cyclic loops 


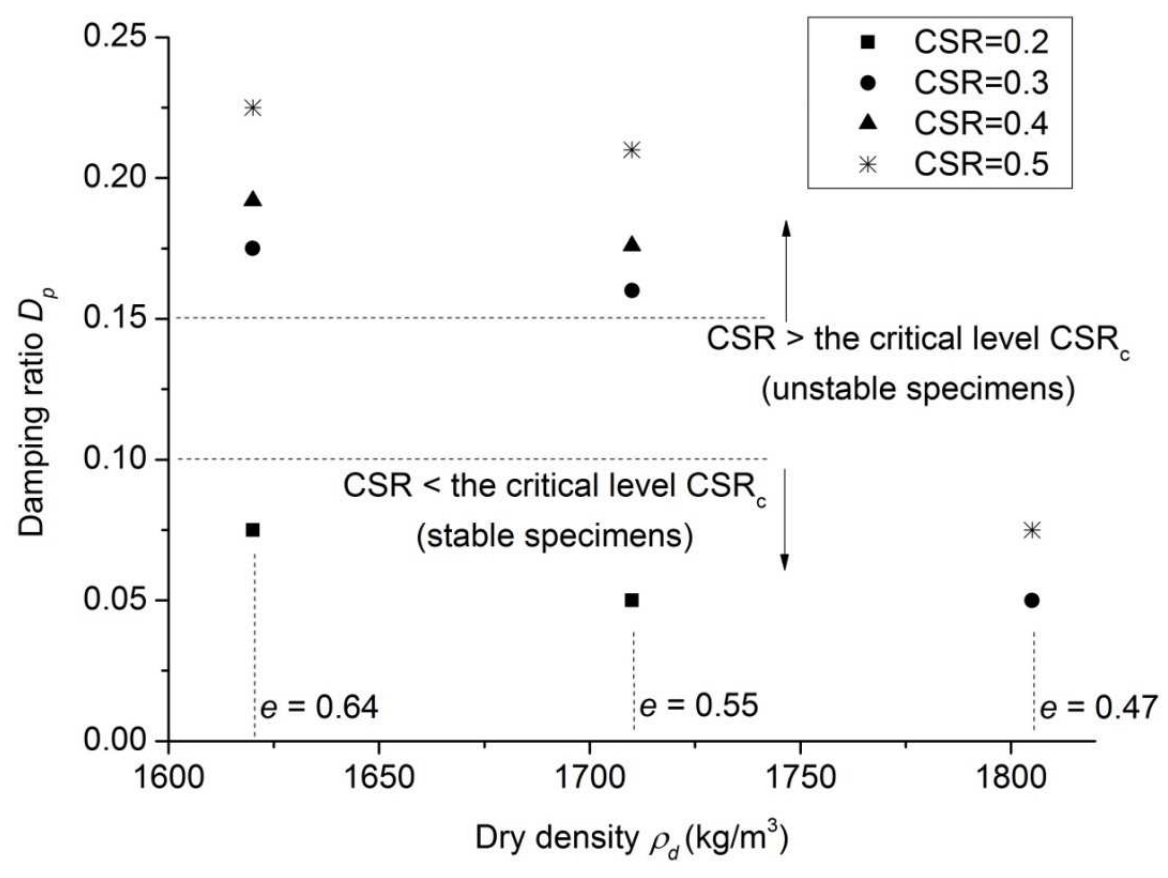

Fig. 9 Variation of $D_{p}$ with different soil density (void ratio) 


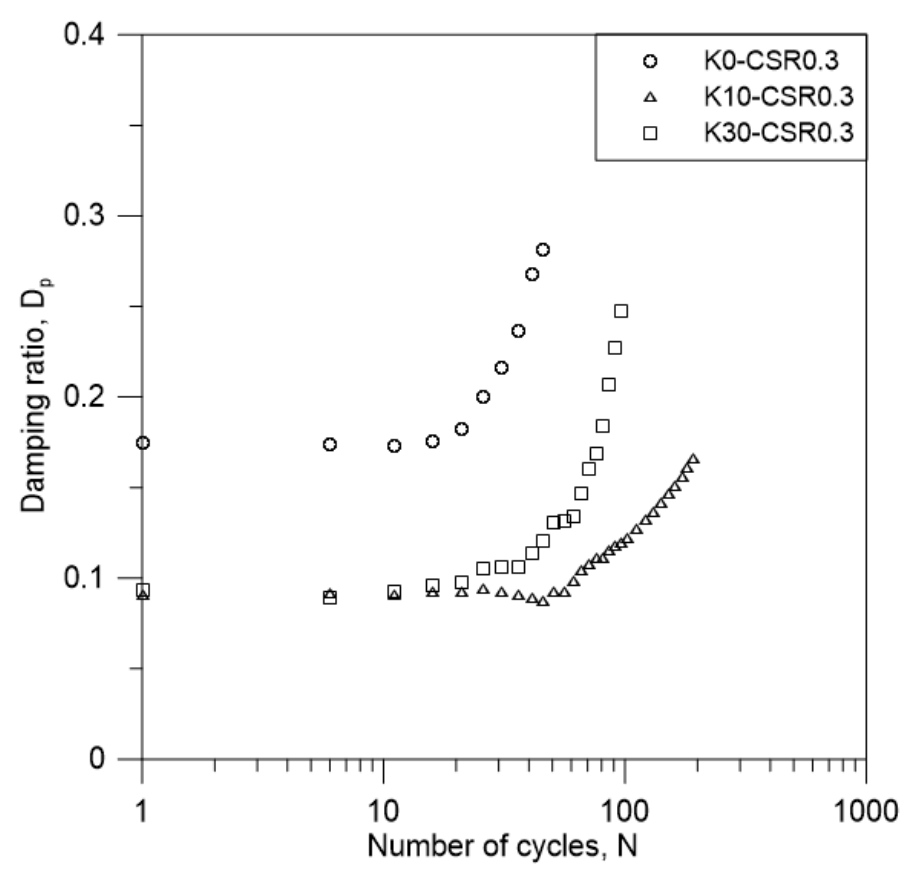

a)

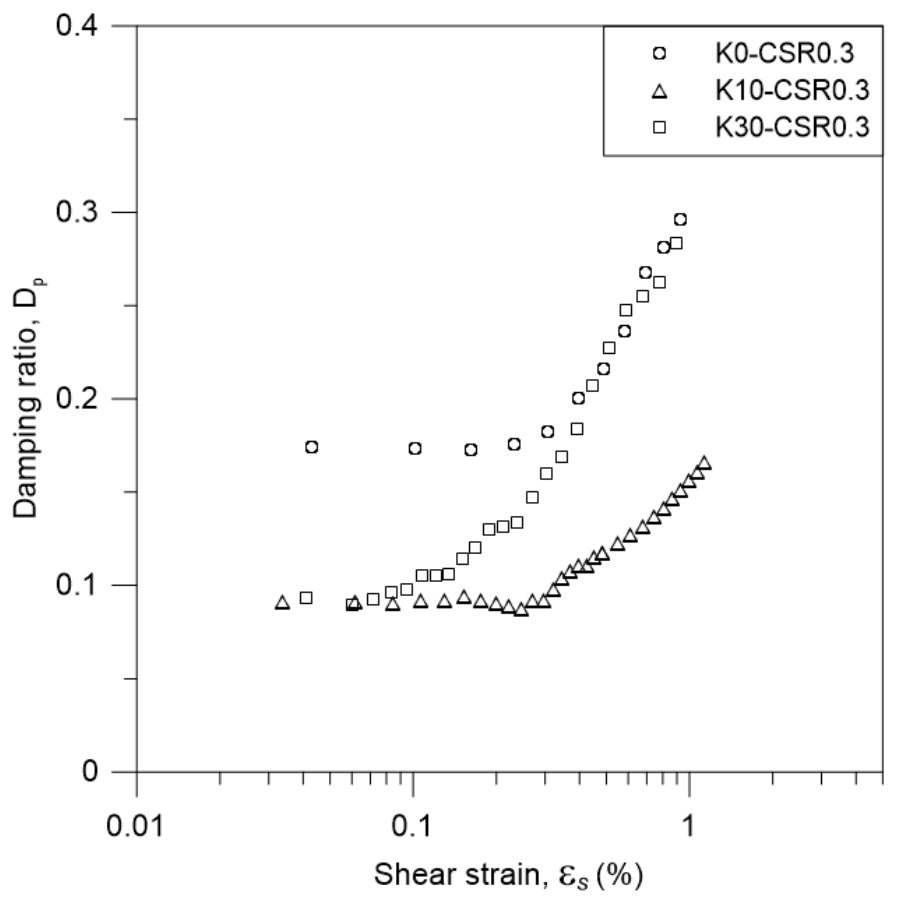

b)

Fig. 10 Influence of kaolin content on damping properties of subgrade soil: a) with number of cycles $N$; and b) with shear strain $\mathcal{E}_{s}$ 


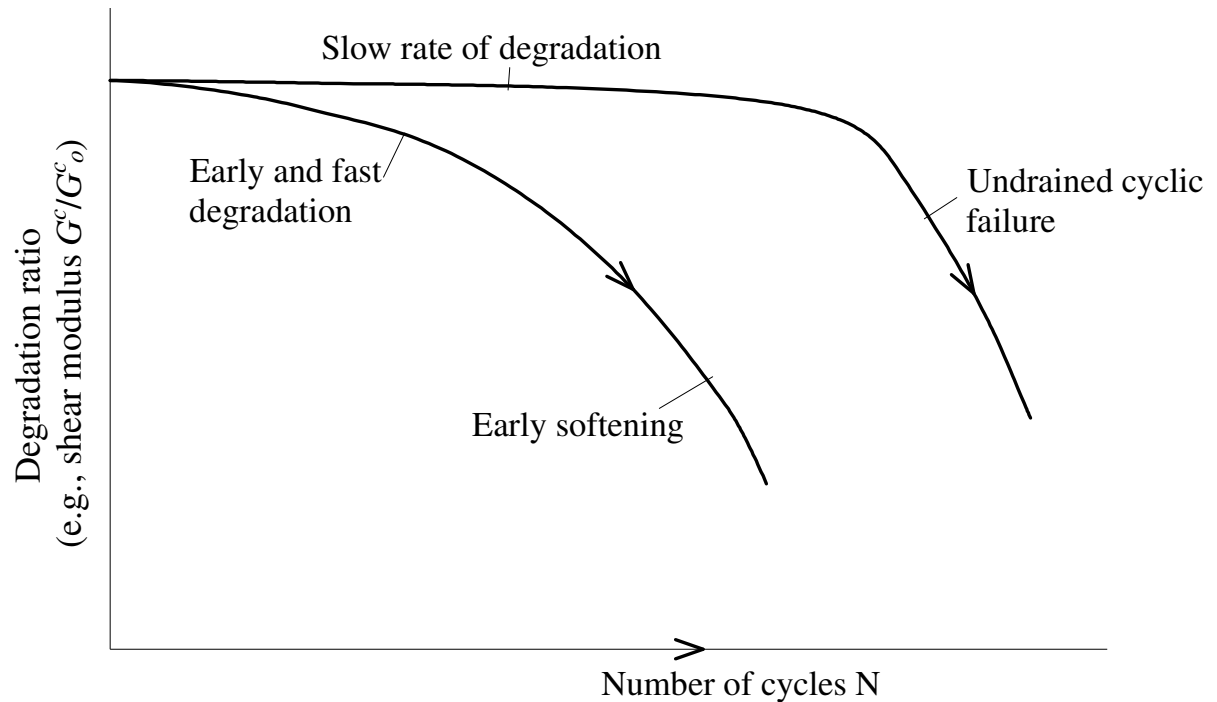

Fig. 11 Two different forms of degradation corresponding to the early softening and undrained cyclic failures of soil when $C S R \geq C S R_{c}$ 


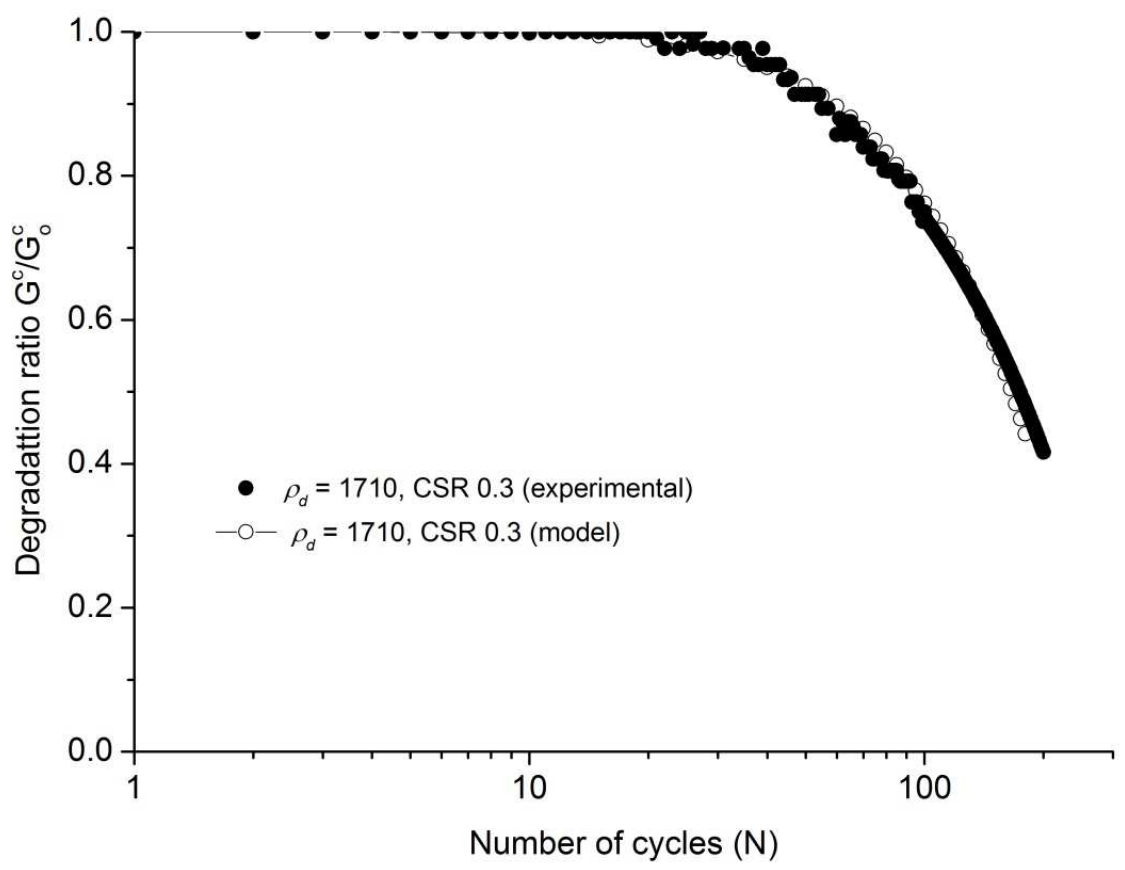

a)

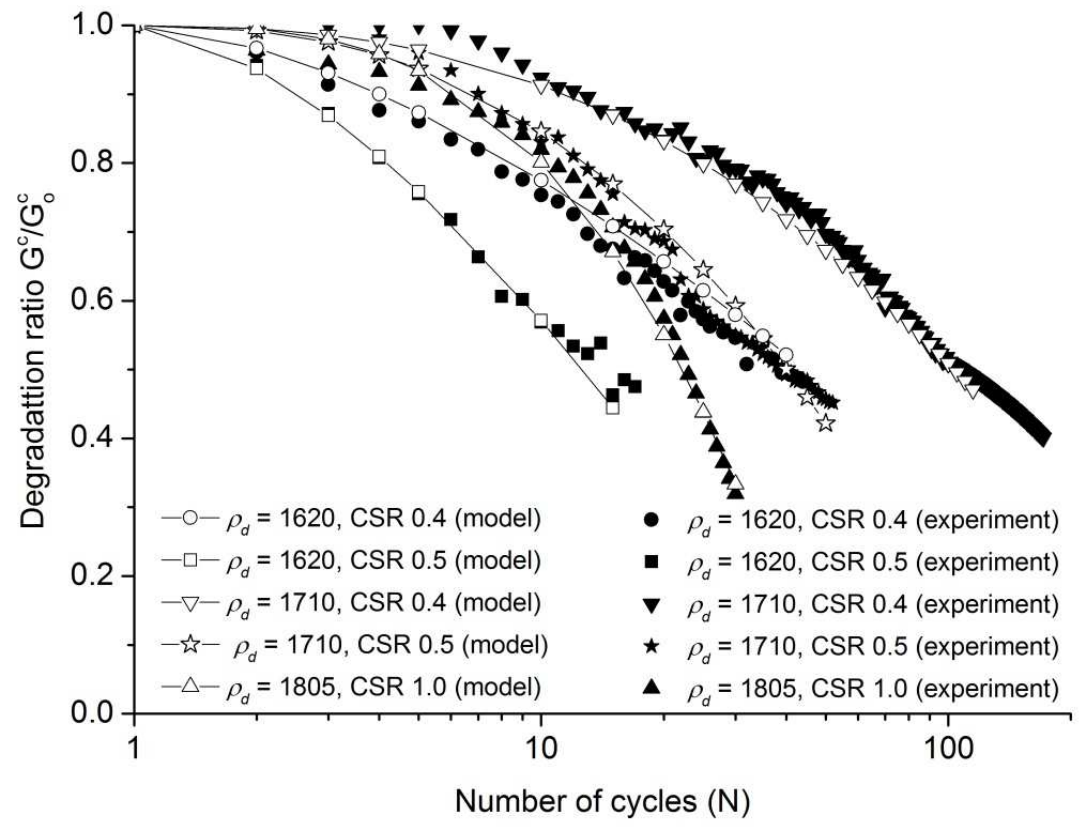

b)

Fig. 12 Empirical estimation of the degradation in shear modulus: a) undrained shear failure; b) early softening; and c) model application to other experimental data (Głuchowski and Sas 2020). 


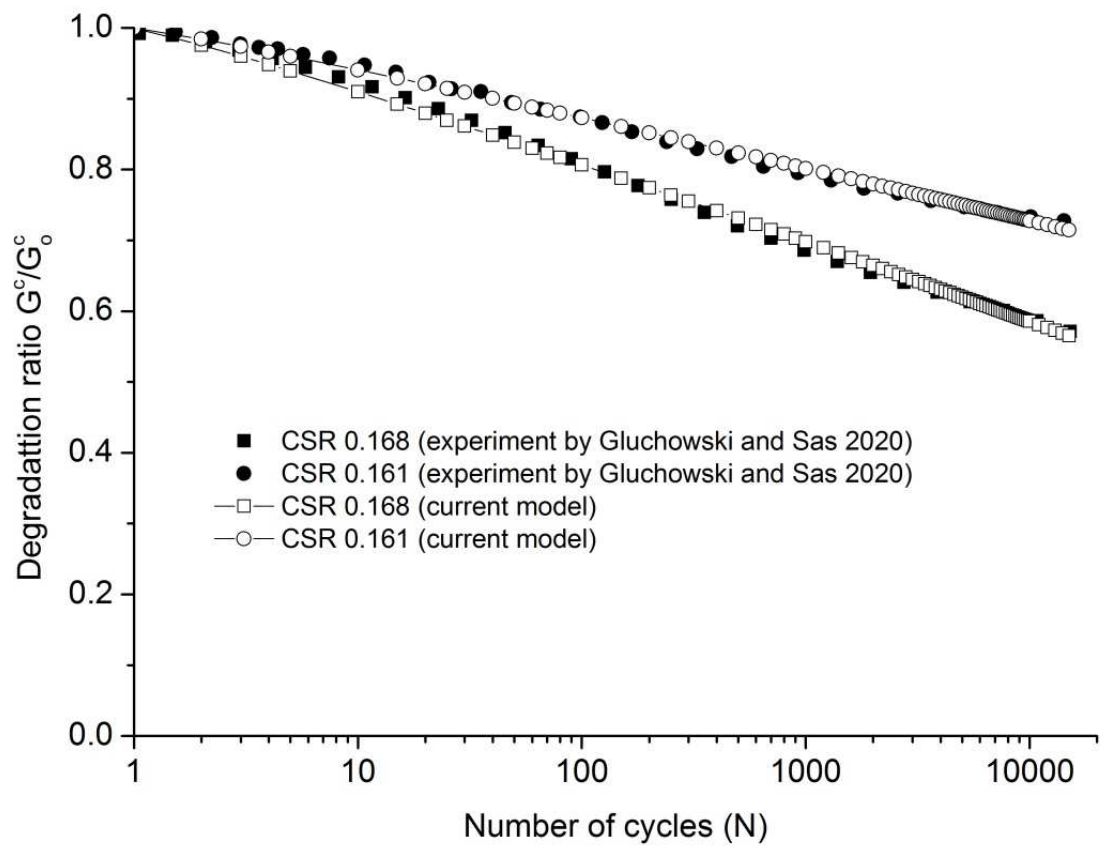

Note: CSR in the original study (Gtuchowski and Sas 2020) is recomputed based on the current definition of CSR (Indraratna et al. 2020a)

c)

Fig. 12 Empirical estimation of the degradation in shear modulus: a) undrained shear failure; b) early softening; and c) model application to other experimental data (Głuchowski and Sas 2020) (continued) 


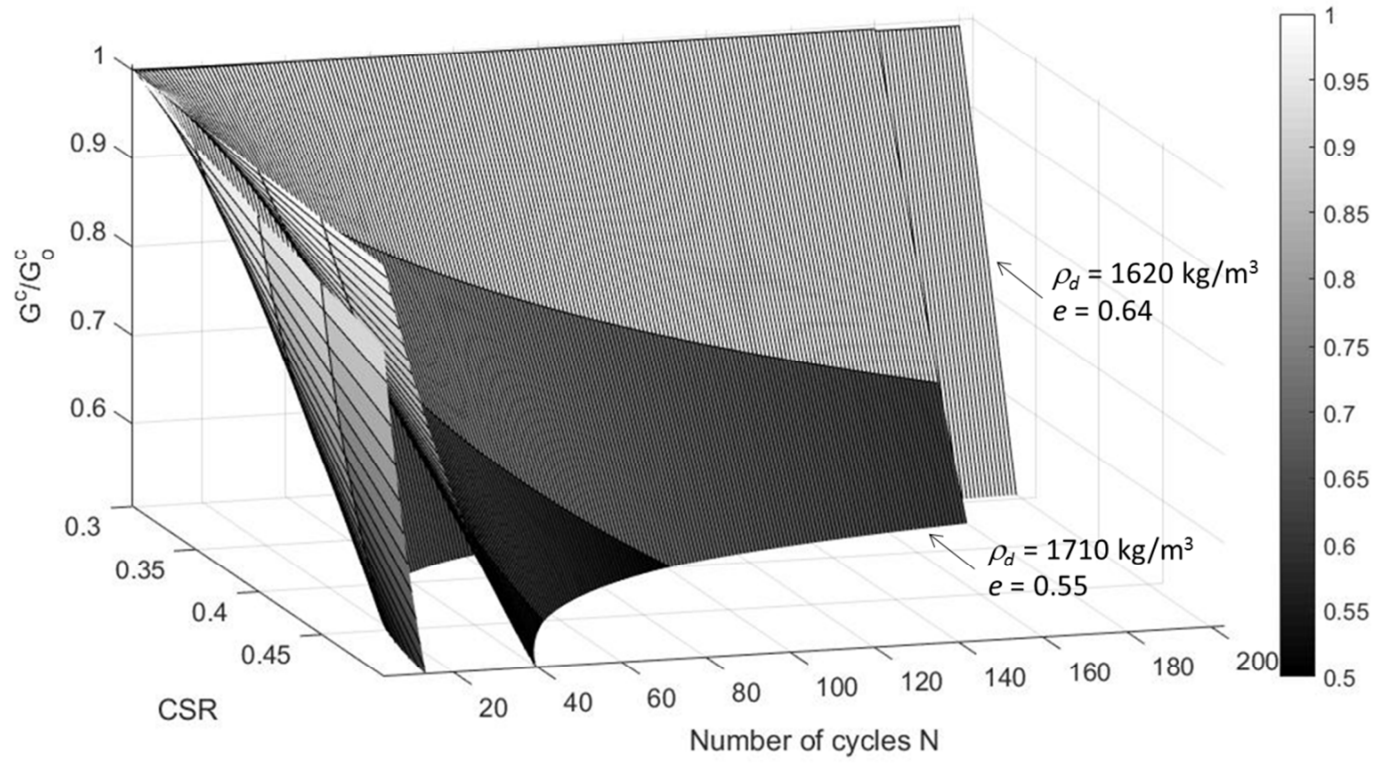

Fig. 13 The early softening surface $\left(G^{c} / G_{o}^{c}\right)$ of subgrade soil subjected to heavy haul rail load (i.e., varying load parameters $C S R$ and $N$ ) 


\section{References}

Arroyo, M., Wood, D.M. and Greening, P.D. 2003. Source near-field effects and pulse tests in soil samples. Géotechnique, 53(3): 337-345. doi: 10.1680/geot.2003.53.3.337.

ASTM D3999 - 91. 2003. Standard test methods for the determination of the modulus and damping properties of soils using the cyclic triaxial apparatus. American Society for Testing and Materials, West Conshohocken, Pa.

Australian Trade Commission 2013. Heavy haul, Intermodal and freight rail.

Cai, Y., Wu, T., Guo, L. and Wang, J. 2018. Stiffness Degradation and Plastic Strain Accumulation of Clay under Cyclic Load with Principal Stress Rotation and Deviatoric Stress Variation. Journal of Geotechnical and Geoenvironmental Engineering, 144(5): 04018021. doi: doi:10.1061/(ASCE)GT.1943-5606.0001854.

D' Elia, B. and Lanzo, G. 1996. Laboratory and field determinations of small-strain shear modulus of natural soil deposits. In Proceedings of the the 11th World Conference on Earthquake Engineering.

Dareeju, B., Gallage, C., Ishikawa, T. and Dhanasekar, M. 2017. Effects of principal stress axis rotation on cyclic deformation characteristics of rail track subgrade materials. Soils and $\quad$ Foundations, $\quad$ 423-438. https://doi.org/10.1016/j.sandf.2017.05.009.

Esmaeili, M., Zakeri, J.A., Ebrahimi, H. and Sameni, M.K. 2016. Experimental study on dynamic properties of railway ballast mixed with tire derived aggregate by modal shaker test. Advances in Mechanical Engineering, 8(5): 1-13.

GDS. 2020. Bender Element System (BEs)-GDS Instruments. https://www.gdsinstruments.com/.

Głuchowski, A. and Sas, W. 2020. Impact of Cyclic Loading on Shakedown in Cohesive 
Soils—Simple Hysteresis Loop Model. Applied Sciences, 10(6): 2029.

Gräbe, P.J. and Clayton, C.R. 2009. Effects of Principal Stress Rotation on Permanent Deformation in Rail Track Foundations. Journal of Geotechnical and Geoenvironmental Engineering, 135(4): 555-565. doi: doi:10.1061/(ASCE)10900241(2009)135:4(555).

Heitor, A., Indraratna, B. and Rujikiatkamjorn, C. 2013. Laboratory study of small-strain behavior of a compacted silty sand. Canadian Geotechnical Journal, 50(2): 179-188. doi: 10.1139/cgj-2012-0037.

Hussien, M.N. and Karray, M. 2015. Shear wave velocity as a geotechnical parameter: an overview. Canadian Geotechnical Journal, 53(2): 252-272. doi: 10.1139/cgj-20140524.

Ichii, K. and Mikami, T. 2018. Cyclic threshold shear strain in pore water pressure generation in clay in situ samples. Soils and Foundations, 58(3): 756-765. doi: https://doi.org/10.1016/j.sandf.2018.01.005.

Indraratna, B., Korkitsuntornsan, W. and Nguyen, T.T. 2020a. Influence of Kaolin content on the cyclic loading response of railway subgrade. Transportation Geotechnics, 22: 100319. doi: https://doi.org/10.1016/j.trgeo.2020.100319.

Indraratna, B., Singh, M., Nguyen, T.T., Leroueil, S., Abeywickrama, A., Kelly, R. and Neville, T. 2020b. A laboratory investigation on fluidization of subgrade under cyclic train loading. Canadian Geotechnical Journal. doi: 10.1139/cgj-2019-0350.

Kallioglou, P., Tika, T. and Pitilakis, K. 2008. Shear Modulus and Damping Ratio of Cohesive Soils. Journal of Earthquake Engineering, 12(6): 879-913. doi: $10.1080 / 13632460801888525$.

KarrayMourad, LefebvreGuy, EthierYannic and BigrasAnnick. 2011. Influence of particle size on the correlation between shear wave velocity and cone tip resistance. Canadian 
Geotechnical Journal, 48(4): 599-615. doi: 10.1139/t10-092.

Kim, U., Kim, D. and Zhuang, L. 2016. Influence of fines content on the undrained cyclic shear strength of sand-clay mixtures. Soil Dynamics and Earthquake Engineering, 83: 124-134. doi: https://doi.org/10.1016/j.soildyn.2016.01.015.

Kokusho, T. and Yoshida, Y. 1997. SPT N-Value and S-Wave Velocity for Gravelly Soils with Different Grain Size Distribution. Soils and Foundations, 37(4): 105-113. doi: https://doi.org/10.3208/sandf.37.4_105.

Konstadinou, M. and Georgiannou, V.N. 2014. Prediction of pore water pressure generation leading to liquefaction under torsional cyclic loading. Soils and Foundations, 54(5): 993-1005. doi: https://doi.org/10.1016/j.sandf.2014.09.010.

Kramer, S.L. 1996. Geotechnical earthquake engineering.Prentice-Hall international series in civil engineering and engineering mechanics. Pearson Education, New Jersey. p.653.

Kulkarni, M.P., Patel, A. and Singh, D.N. 2010. Application of shear wave velocity for characterizing clays from coastal regions. KSCE Journal of Civil Engineering, 14(3): 307-321. doi: 10.1007/s12205-010-0307-1.

Lee, J.-S. and Santamarina, J.C. 2005. Bender Elements: Performance and Signal Interpretation. Journal of Geotechnical and Geoenvironmental Engineering, 131(9): 1063-1070. doi: doi:10.1061/(ASCE)1090-0241(2005)131:9(1063).

Li, D. and Selig, E.T. 1996. Cumulative Plastic Deformation for Fine-Grained Subgrade Soils. Journal of Geotechnical Engineering, 122(12): 1006-1013. doi: doi:10.1061/(ASCE)0733-9410(1996)122:12(1006).

Long, M. and Donohue, S. 2010. Characterization of Norwegian marine clays with combined shear wave velocity and piezocone cone penetration test (CPTU) data. Canadian Geotechnical Journal, 47(7): 709-718. doi: 10.1139/t09-133.

Mamou, A., Powrie, W., Priest, J.A. and Clayton, C. 2017a. The effects of drainage on the 
behaviour of railway track foundation materials during cyclic loading. Géotechnique, 67(10): 845-854. doi: 10.1680/jgeot.15.P.278.

Mamou, A., Priest, J.A., Clayton, C.R.I. and Powrie, W. 2017b. Behaviour of saturated railway track foundation materials during undrained cyclic loading. Canadian Geotechnical Journal, 55(5): 689-697. doi: 10.1139/cgj-2017-0196.

Navaratnarajah, S.K. and Indraratna, B. 2017. Use of Rubber Mats to Improve the Deformation and Degradation Behavior of Rail Ballast under Cyclic Loading. Journal of Geotechnical and Geoenvironmental Engineering, 143(6): 04017015. doi: doi:10.1061/(ASCE)GT.1943-5606.0001669.

Nguyen, T.T. and Indraratna, B. 2020a. A coupled CFD-DEM approach to examine the hydraulic critical state of soil under increasing hydraulic gradient. ASCE International Journal of Geomechanics, 20(9): 04020138-1:15. doi: https://doi.org/10.1061/(ASCE)GM.1943-5622.0001782.

Nguyen, T.T. and Indraratna, B. 2020b. The energy transformation of internal erosion based on fluid-particle coupling. Computers and Geotechnics, 121: 103475. doi: https://doi.org/10.1016/j.compgeo.2020.103475.

Nguyen, T.T. and Indraratna, B. 2021. Rail track degradation under mud pumping evaluated through site and laboratory investigations. International Journal of Rail Transportation: 1-28. doi: https://doi.org/10.1080/23248378.2021.1878947.

Nguyen, T.T., Indraratna, B., Kelly, R., Phan, N.M. and Haryono, F. 2019. Mud pumping under railtracks: Mechanisms, Assessments and Solutions. Australian Geomechanics Journal, 54(4): 59-80.

Ogino, T., Kawaguchi, T., Yamashita, S. and Kawajiri, S. 2015. Measurement deviations for shear wave velocity of bender element test using time domain, cross-correlation, and frequency domain approaches. Soils and Foundations, 55(2): 329-342. doi: 
https://doi.org/10.1016/j.sandf.2015.02.009.

Park, D. and Stewart, H.E. 2001. Suggestion of empirical equations for damping ratio of plastic and non-plastic soils based on the previous studies. In Proceedings of the 4th International Conference on Recent Advances in Geotechnical Earthquake Engineering and Soil Dynamics.17. University of Missouri--Rolla pp. 1.21.

Priest, J.A., Powrie, W., Yang, L., Grabe, P.J. and Clayton, C.R.I. 2010. Measurements of transient ground movements below a ballasted railway line. Géotechnique, 60(9): 667-677. doi: 10.1680/geot.7.00172.

Railway Gazette International. 2016. BHP breaks its own 'heaviest train' record. https://www.railwaygazette.com/news/bhp-breaks-its-own-heaviest-trainrecord/29603.article.

Rollins, K.M., Evans, M.D., Diehl, N.B. and III, W.D.D. 1998. Shear Modulus and Damping Relationships for Gravels. Journal of Geotechnical and Geoenvironmental Engineering, 124(5): 396-405. doi: doi:10.1061/(ASCE)1090-0241(1998)124:5(396).

Shibuya, S., Mitachi, T., Fukuda, F. and Degoshi, T. 1995. Strain Rate Effects on Shear Modulus and Damping of Normally Consolidated Clay. Geotechnical Testing Journal, 18(3): 365-375. doi: 10.1520/gtj11005j.

Sun, J.I., Golesorkhi, R. and Seed, H.B. 1988. Dynamic moduli and damping ratios for cohesive soils. In Earthquake Engineering Research Center. College of Engineering, University of California at Berkeley.

Truong, M.H., Indraratna, B., Nguyen, T.T., Carter, J. and Rujikiatkamjorn, C. 2021. Analysis of undrained cyclic response of saturated soils. Computers and Geotechnics, 134: 104095. doi: https://doi.org/10.1016/j.compgeo.2021.104095.

Viggiani, G. and Atkinson, J.H. 1995. Interpretation of bender element tests. Géotechnique, 45(1): 149-154. doi: 10.1680/geot.1995.45.1.149. 
Vucetic, M. and Dobry, R. 1991. Effect of Soil Plasticity on Cyclic Response. Journal of Geotechnical Engineering, 117(1): 89-107. doi: doi:10.1061/(ASCE)07339410(1991)117:1(89).

Vucetic, M., Lanzo, G. and Doroudian, M. 1998a. Damping at small strains cyclic simple shear test. Journal of Geotechnical \& Geoenvironmental Engineering, 124(7): 585594.

Vucetic, M., Lanzo, G. and Doroudian, M. 1998b. Effect of The Shape of Cyclic Loading on Damping Ratio at Small Strains. Soils and Foundations, 38(1): 111-120. doi: https://doi.org/10.3208/sandf.38.111.

Wang, Y.H., Lo, K.F., Yan, W.M. and Dong, X.B. 2007. Measurement Biases in the Bender Element Test. Journal of Geotechnical and Geoenvironmental Engineering, 133(5): 564-574. doi: doi:10.1061/(ASCE)1090-0241(2007)133:5(564). 